\title{
Fretting Fatigue Damage Nucleation and Propagation Lifetime Using a Central Point Movement of Power Spectral Density
}

\author{
Thanh Q. Nguyen $\mathbb{D}^{1},{ }^{1}$ Hieu C. Doan, ${ }^{2}$ Luan C. Vuong, ${ }^{1}$ H. Nguyen-Xuan, ${ }^{3}$ and Nhi K. Ngo ${ }^{1}$ \\ ${ }^{1}$ Laboratory of Applied Mechanics (LAM), Ho Chi Minh City University of Technology, \\ VNU-HCM, Ho Chi Minh City 740030, Vietnam \\ ${ }^{2}$ Ho Chi Minh City Department of Transportation and Communication, Ho Chi Minh City 710100, Vietnam \\ ${ }^{3}$ CIRTECH Institute, Ho Chi Minh City University of Technology (HUTECH), Ho Chi Minh City 710100, Vietnam
}

Correspondence should be addressed to Thanh Q. Nguyen; nqthanh@hcmut.edu.vn

Received 19 October 2019; Accepted 26 December 2019; Published 22 January 2020

Academic Editor: Angelo Marcelo Tusset

Copyright ( $) 2020$ Thanh Q. Nguyen et al. This is an open access article distributed under the Creative Commons Attribution License, which permits unrestricted use, distribution, and reproduction in any medium, provided the original work is properly cited.

\begin{abstract}
This paper presents a new perception in evaluating fretting fatigue damage nucleation and propagation lifetime under periodically forced circulation. A new approach, which is proposed in this paper, is to measure the change of the central point of power spectral density (CP-PSD) in different structural stiffness degradation stages. A notable aspect of this study lies in the combination between vibration amplitude and forced frequency of the fatigue-causing factors in beam structures. Additionally, it is found that randomization of the first phase from 0 to $2 \pi$ yields more accurate modelling of the fatigue phenomenon. Results show that the CP-PSD parameter is significantly more sensitive compared to the regularly damage-evaluating parameters such as natural frequency, eigenvalues, or stress value. This reflects different levels of fatigue cycle effect on the structure in the experiment. At the same time, CP-PSD also categorizes the degradation level on different points on the structure under the periodically forced circulation. In addition, this paper also quantifies the relation between the changes of CP-PSD and each fatigue state. Results of this research will be a reference source to evaluate the lifespan of the structure by experimental methods.
\end{abstract}

\section{Introduction}

The experiment on the effect of fatigue on material and working capacity of the structure is an important topic. Fatigue affects significantly the design and operation of the structure. In particular, the load-bearing parts of the structure have circulation because they are always under the periodical or changeable load. Practical structures are always designed to work within a certain lifespan. If lifespan is too short, a structure will not satisfy the requirements given, indicating the design does not meet the requirements or the operation goes beyond the requirements. If lifespan is too long, the structure will exceed in size or the weight will not meet the design. All of the mentioned scenarios show that the fatigue evaluation helps forecast the lifespan as well as ensures the working capacity of the structure. Although this evaluation is not a new topic, we still encounter various difficulties when it comes to practical application.
Theoretically, fatigue is part of duration science, which focuses on the characteristics of the material as well as structure form under the changeable load over time. According to Burdekin and Stone [1], Hutchinson [2], Rice and Rosengren [3], and Shih [4], when evaluating effect of numerous factors on the structure, they found that these factors gradually degraded the underload material over time. Additionally, these authors also proposed the evaluation method and technical solution to enhance the duration of the structure impacted by the effect. Based on the reality, we can categorize fatigue into 2 forms: short-term fatigue (temporary) and long-term fatigue (permanent). When considering the effect of short-term fatigue on the structure, Pereira et al. [5] and Kumar et al. [6] concluded that it was the first condition for the crack's development in the structure. However, it did not affect the load-bearing capacity of the structure yet. If the fatigue continued regardless of time and intensity, short-term fatigue would transform 
into long-term fatigue. This was the first experiment for short-term fatigue to happen when stress cycle and amplitude of the vibration were low. Conversely, long-term fatigue has high-stress period, high amplitude of vibration, and wide exposure surface, which harms the surface of the structure seriously and at the same time facilitates the development and expansion of potential defects in the structure. According to Pereira et al. [7], Yue and Abdel Wahab [8-10], and Vingsbo and Söderberg [11], under the effect of long-term fatigue, the exposure area would yield in serious damage that directly affected the operation of the structure. If fatigue kept working, the structure would be damaged or completely destroyed. Nowadays, due to the significant effect of fatigue on projects, machine, and device, the fatigue theory has gained many successful achievements and new steps in comparison to the very first days. Numerous research studies have offered new parameters in evaluating and observing fatigue. This theoretical foundation has been widely applied in evaluating the duration of machine construction as well as structures that bear the load and it make changed stress. Recently, Bhatti and Abdel Wahab and their coworkers have gained the most successful achievements using the fatigue effect of the structure as an effective method to evaluate the degradation level of structures $[12,13]$. They also proposed many approaches to detect and observe defects in structures. However, it is clear that the results of above experiments were only sensitive with simulated or in-house experiment. They did not show clearly the application and how they would define the lifespan of the structure. To evaluate the operation of the structure under the fatigue effect by practical methods, some experiments usually based on the physical parameters such as Findley stress [14, 15], McDiarmid [16] with cycle multiaxial fatigue, Lykins et al. [17] with shear stress-based parameter, Fatemi distortion parameter [18], elastic deformation energy of Smith et al. [19], Szolwinski [20] with fretting fatigue crack formation, and stress mode shapes of Zhou [21-23] were carried out. Therein, the parameters extracted from the deformation value are used more often as the relation of deformation stress is the foundation for evaluating fatigue. However, in reality, fatigue affected by the vibration of the structure over time accounts for the majority. In other words, vibration is the cause of fatigue and degradation of the structure over time. The use of the vibration method in studying fatigue encounters many difficulties due to the complex behavior of both structures and affecting factors. There are many types of vibrations that affect the structure at the same time: predestined, forced, free, or mixed vibration (the combination of different vibrations), which make the behavior of the structure complicated. These are also main causes that make fatigue common in reality, but the number of research studies applied to solve this issue is relatively few.

The current research improves upon shortcomings of previous studies such as either separately evaluating inducing fatigue-causing factors as vibration amplitude, forced frequency, and first phase or using only numerical simulations to evaluate fatigue from multiple factors by the computer. A new paradigm is presented in investigating the decline of beams under harmonic constraining force through vibration analysis. The subject of investigation-beam-type models-is at the core of various real-life structures; yet, despite being a topic of major interest, it is rarely researched thoroughly due to the extensive and complicated fieldwork required. Therefore, this research highlights the characteristics of beam structures under the fatigue phenomenon. Through the three mentioned factors causing fatigue, the new CP-PSD parameter is presented. Using vibration measurements collected from accelerometers, we composed the signal's power spectrum across various phases (periods) of fatigue on the beam. Research demonstrated that changes in the CP-PSD at each phase of fatigue can be used as a basis of evaluating decline in beams over time. Furthermore, shifts in CP-PSD are found to be significantly more sensitive than previous parameters such as natural frequency, eigenvalues, or stress value. CP-PSD reflects various degrees of decline in the beam under the effect of the periodical fatigue propagation during experimentation. This shall be the basis of applying fatigue to determine structures' lifespans through experiments.

\section{Theoretical Background}

The experiment model investigates fatigue generated on the beam by harmonic constraining force. Thanks to this theory, we propose the application of the harmonic function as the input for fatigue process (vibration amplitude and forced frequency) of Hysteresis process. This is the basis to define the fatigue period as well as degradation level over time on the experimented beam.

2.1. The Beam Model under Harmonic Constraining Force. We consider the beam model simulated as a single rest beam with 2 tips as shown in Figure 1. It bears the effect of harmonic constraining force $P(t)=Q \sin (\Omega t+\theta)$, wherein $Q$ is the amplitude of forced circulation on the beam and $\Omega$ is the trigger frequency on the beam. The vibrating function is expressed by equation (1). When considering the geometry characteristic of the beam structure, under the harmonic constraining force, the beam reflects the basis physical behavior of the bending vibration.

The expression of the beam bearing the bending is given as

EJ $\frac{\partial w^{4}(x, t)}{\partial x^{4}}+\rho \frac{\partial w^{2}(x, t)}{\partial t^{2}}+2 \mu \omega_{b} \frac{\partial w(x, t)}{\partial t}=\delta(x-c t) P(t)$.

The boundary and first conditions are given by

$$
\begin{aligned}
w(0, t) & =0, \\
w(l, t) & =0, \\
\left.\frac{\partial^{2} w(x, t)}{\partial x^{2}}\right|_{x=0} & =0, \\
\left.\frac{\partial^{2} w(x, t)}{\partial x^{2}}\right|_{x=l} & =0, \\
w(x, 0) & =0 ; \\
\left.\frac{\partial w(x, t)}{\partial^{t}}\right|_{t=0} & =0,
\end{aligned}
$$




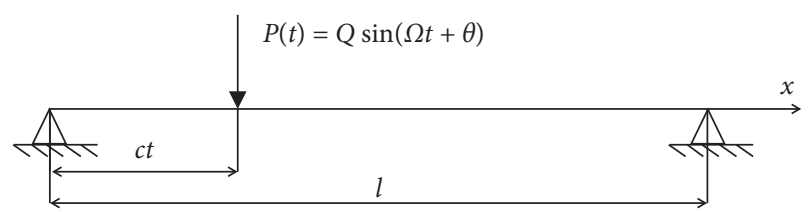

FIgURE 1: The simple rest beam model with moving load.

where $x$ is the coordinate of the concentrated force and is defined by the origin coordinate of beam; $t$ is the effect time of load on the beam and defined from the start of the load until the period is changed or finished completely; $w(x, t)$ is the deflection of the beam at $x$ and time $t ; c$ is the load velocity; $\rho$ is the density of the beam of a certain length; $\omega_{b}$ is the damper frequency of the beam; $P(t)$ is the magnitude of the affecting band; $l$ is the length of the beam; and $\delta(\cdot)$ is the fatigue function on the beam. The $\delta(\cdot)$ function on the beam depends on 2 factors which are position and velocity of the force. The function is defined by the following equation:

$$
\int_{b}^{a} \delta(x-\varepsilon) F(x) \mathrm{d} x= \begin{cases}0, & \varepsilon<a<b, \\ F(\varepsilon), & a<\varepsilon<b, \\ 0, & a<b<\varepsilon .\end{cases}
$$

To solve equation (1), we use Galerkin method which is one of the most appropriate methods in solving variable problems relating to structural dynamics. The root of equation (1) is chosen as a variable dissociation as equation (4). Therein, $\phi_{j}(x)$ are linearly independent functions that satisfy the boundary condition of the model structure, and $q_{j}(t)$ function varies over time and is defined by the integral of normal partial derivatives as follows:

$$
\int_{0}^{l}\left\{L\left[\sum_{j=1}^{n} q_{j}(t) \phi_{j}(x)\right]-p(x, t)\right\} \phi_{j}(x) \mathrm{d} x=0 .
$$

With the boundary condition equation (2a), we choose $\phi(x)=\sin (j \pi x / l)$ and integral of terms from 0 to $l$, and the variable Fourier integral in Sin-type is expressed as follows:

$$
\begin{aligned}
w(x, t) & =\frac{2}{l} \sum_{j=1}^{\infty} W(j, t) \sin \frac{j \pi x}{l}, \\
W(j, t) & =\int_{0}^{l} w(x, t) \sin \frac{j \pi x}{l} \mathrm{~d} x, \quad j=1,2,3, \ldots
\end{aligned}
$$

Substituting equation (5b) into equation (4), one writes

$$
\begin{aligned}
& \frac{j^{4} \pi^{4}}{l^{4}} \operatorname{EJW}(j, t)+\rho \ddot{W}(j, t)+2 \mu \omega_{b} \dot{W}(j, t) \\
& =\int_{0}^{l} \partial(x-c t) P(t) \sin \frac{j \pi x}{l} \mathrm{~d} x \\
& =>\ddot{W}(j, t)+2 \omega_{b} \dot{W}(j, t)+\omega_{j}^{2} W(j, t)=\frac{P(t)}{\rho} \sin j \omega_{c} t .
\end{aligned}
$$

The specific vibration frequency of the structure according to the $j$ th mode is defined by the following equation:

$$
\omega_{j}^{2}=\frac{j^{4} \pi^{4}}{l^{4}} \frac{\mathrm{EJ}}{\rho}, \quad j=1,2, \ldots, n,
$$

and the velocity frequency is

$$
\omega_{c}=\frac{\pi c}{l} .
$$

To solve equation (6), we use the Laplace-Carson integral transformation method. Multiplying each term with $e^{-P t}$, integrating by $t$ the terms from $[0, \infty]$, and then multiplying all by $P$, which is the variable of the complex plane

$$
\begin{aligned}
& P^{2} W^{*}(j, P)+2 \omega_{b} P W^{*}(j, P)+\omega_{j}^{2} W^{*}(j, P) \\
& =P \int_{0}^{\infty} \frac{F(t)}{\rho} \sin j \omega_{c} t e^{-p t} \mathrm{~d} t,
\end{aligned}
$$

with

$$
\begin{gathered}
W^{*}(j, P)=P \int_{0}^{\infty} W(j, t) e^{-p t} \mathrm{~d} t, \\
W(j, t)=\frac{1}{2 \pi i} \int_{a_{0}-i \infty}^{a_{0}+i \infty} e^{t P} \frac{W^{*}(j, P)}{P} \mathrm{~d} P, \quad j=1,2, \ldots, n .
\end{gathered}
$$

For the experimental model, one uses $P(t)=Q$ $\sin (\Omega t+\theta)$ function, and equation (6) turns into

$$
\begin{aligned}
& p^{2} W^{*}(j, P)+2 \omega_{b} P W^{*}(j, P)+\omega_{j}^{2} W^{*}(j, P) \\
& =P \frac{Q}{2 \rho} \int_{0}^{\infty}\left[\cos \left(\left(\Omega-j \omega_{c}\right)+\theta\right)-\cos \left(\left(\Omega+j \omega_{c}\right)+\theta\right)\right] e^{-p t} \mathrm{~d} t .
\end{aligned}
$$

The manuscript proposes the limitation and complexity level of harmonic force on the beam structure by the fatigue phenomenon, the first phase $\theta_{0}=0$. However, when conducting the experiment, it was hard to define the first phase of the circulation, so we suggest to expand this first phase for the case $\theta$ as a random variable in the range from 0 to $2 \pi$. Hence, the solving process of equation (11) is simplified, and the first phase $\theta$ will not be fixed as in the research manuscript.

Setting $r_{1}=\Omega+j \omega_{c}$ and $r_{2}=\Omega-j \omega_{c}$, we have

$$
\begin{aligned}
& W^{*}(j, P)=\frac{Q}{2 \rho}\left[P^{2} \cos \theta\left(\frac{1}{P^{2}+r_{2}^{2}}-\frac{1}{P^{2}+r_{1}^{2}}\right)\right. \\
& \left.-p \sin \theta\left(\frac{r_{2}}{P^{2}+r_{2}^{2}}-\frac{r_{1}}{P^{2}+r_{1}^{2}}\right)\right] \frac{1}{\left(P+\omega_{b}\right)^{2}+\omega_{d j}^{2}} .
\end{aligned}
$$

When doing the reverse transformation, we have the original function $W(j, t)$, and adding it into equation (10a), one writes 


$$
\begin{aligned}
w(x, t)= & \frac{Q}{l \rho} \sum_{j=1}^{\infty}\left[\cos \theta\left(A\left(r_{2}\right)-A\left(r_{1}\right)\right)-\sin \theta\left(r_{2} B\left(r_{2}\right)\right.\right. \\
& \left.\left.-r_{1} B\left(r_{1}\right)\right)\right] \sin \frac{j \pi x}{l}
\end{aligned}
$$

with

$$
\begin{aligned}
B(r)= & \frac{1}{\left(\omega_{j}^{2}-r^{2}\right)^{2}+4 \omega_{b}^{2} r^{2}}\left[\frac{\omega_{j}^{2}-r^{2}}{r} \sin r t\right. \\
& -\frac{\omega_{d j}^{2}-\omega_{b}^{2}-r^{2}}{\omega_{d j}} e^{-\omega_{b} t} \sin \omega_{d j} t \\
& \left.-2 \omega_{b}\left(\cos r t-e^{-\omega_{b} t} \cos \omega_{d j} t\right)\right] .
\end{aligned}
$$

2.2. Generating the Fatigue Model on the Beam. In this research, the manuscript uses the fatigue bending method which shows the changeable relation between stress and the relative stress periods. The changeable stress is the highest stress or stress amplitude. In case of tangential stress, if $\sigma_{\max }$ is the stress that changes periodically and has the highest value, $\sigma_{a}$ will be the highest stress amplitude, and if $N$ is the stress period, the classic form of fatigue bending will be expressed as depicted in Figure 2.

Depending on the data processing method in practice, so far, we have had lots of mathematical equations to express the fatigue bending according to equations (15)-(17). In this work, we used the relation bending of stresstransformation to express the relation between fatigue periods and the degradation of the beam is as follows:

$$
\begin{aligned}
a^{\sigma_{a}} \cdot N & =C, \\
\sigma_{a} \cdot N^{d} & =C_{1}, \\
\left(\sigma_{a}-\sigma_{r}\right)(N-B)^{m} & =C_{2},
\end{aligned}
$$

in which $a, d, m, B, C, C_{1}$, and $C_{2}$ are the parameters of the expression: $\sigma_{a}$ is the stress amplitude; $\sigma_{r}$ is the fatigue limit of the material in stress period $r$. Then, the stress period is the shortest time for the repeat of the first initial values. The stress period symbol is $T$ and featured by 3 factors: alternating stress amplitude (see equation (18)), mean stress given in equation (19), and stress ratio given in equation (20).

$$
\begin{aligned}
\sigma_{a} & =\frac{\sigma_{\max }-\sigma_{\min }}{2}, \\
\sigma_{m} & =\frac{\sigma_{\max }+\sigma_{\min }}{2}, \\
r & =\frac{\sigma_{\min }}{\sigma_{\max }} .
\end{aligned}
$$

Practically, the force-bearing period of the hysteresis process includes symmetric stress cycle (see Figure 3),

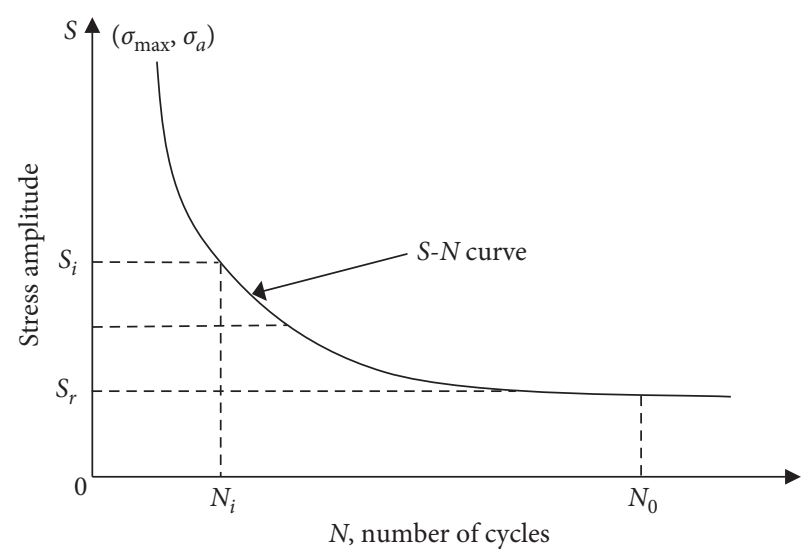

FIgUre 2: Fatigue bending.

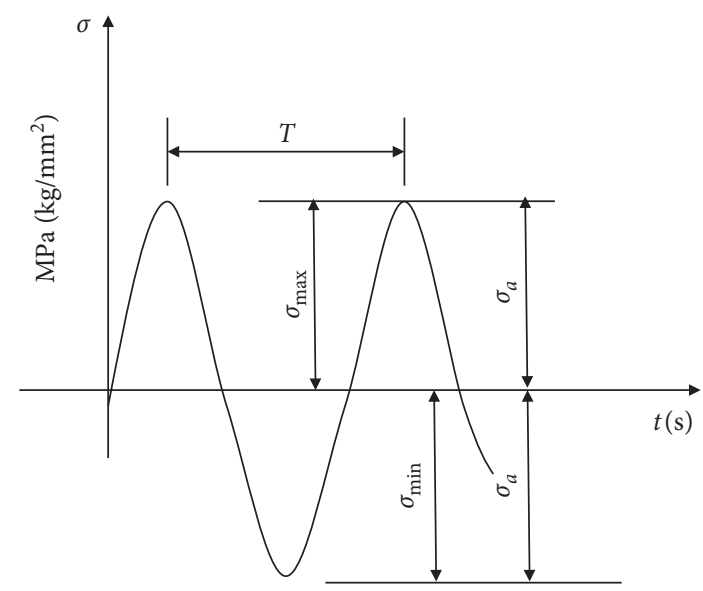

FIGURE 3: Symmetric stress period $\left(\sigma_{m}=0 ; r=-1\right)$.

asymmetric stress cycle as shown in Figure 4, and random stress cycle as depicted in Figure 5.

If $r=-1$ or $r=1$, then the stress cycle is symmetrical. Conversely, if $r=0$, then the stress period is a dynamic form. The asymmetry of the stress period impacts significantly on the fatigue resistance of the material. In reality, the manuscript focuses on conducting research about fatigue destruction for case $r=-1$ and $r=0$ or $r=1$ and $r=0$. It means the fatigue limit of the material, in this case, is given with the symbol $\sigma_{r}=\sigma-1$ and $\sigma_{r}=\sigma_{0}$, respectively. If we apply equation (12) into the beam model in the experiment, under the harmonic constraining force, $P(t)=Q \sin (\Omega t+\theta)$ (the vibration amplitude is $Q$ and vibration frequency is $\Omega$ ). Figures 6(a) and 6(b) show the vibration amplitude $Q$ and $Q_{1}$ $\left(Q_{1}=1.5 Q\right)$ and vibration frequency $\Omega$ and $\Omega_{1}\left(\Omega_{1}=1.5 \Omega\right)$ by the harmonic constraining force $P(t)=Q_{1} \sin (\Omega t+\theta)$.

2.3. A New Parameter in Evaluating the Shift of CP-PSD. In structural dynamics, the stimulating force and response of the system are considered as the input and output of the system. Figure 6 shows that, to evaluate the degradation over time of the structure, what needed is a parameter that is sensitive enough. This parameter can define the change in the structure from one fatigue period to another. Traditional 


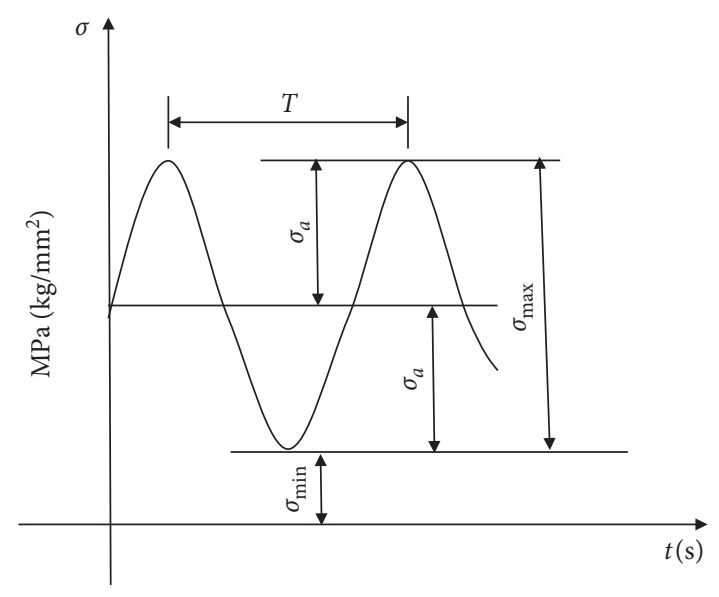

Figure 4: Asymmetric stress period $\left(\sigma_{m} \neq 0 ; r>0\right)$.

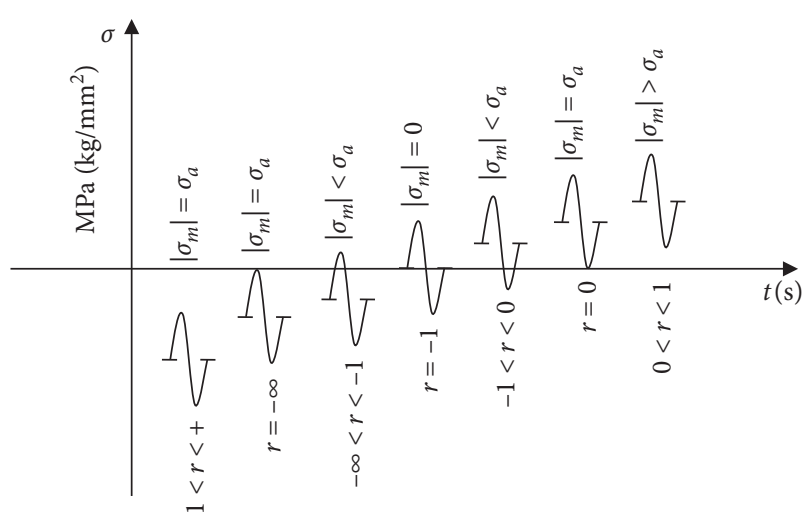

FIGURE 5: Different forms of stress period.

methods are not optimal solutions to this problem. We found that the Fourier transforms over time combined with the signal function from equation (13) could generate the relation between amplitude, forced frequency of the fatigue, and degradation level. Hence, by the PSD of the obtained signal from stimulating the fatigue on the beam, we could evaluate the difference according to Figure 6. The relation between the response of the system and stimulating force is demonstrated as follows:

$$
\begin{aligned}
w(t) & =\int_{-\infty}^{t} h(t-\tau) P(\tau) \mathrm{d} \tau, \\
W(\omega) & =H(\omega) F(\omega),
\end{aligned}
$$

in which the frequency response function $H(\omega)$ and function $F(\omega)$ are the Fourier transform of the response function $h(t)$ and the stimulating force function $P(t)=Q \sin (\Omega t+\theta)$ at the $\tau$ time point ( $\Delta t$ from time $t$ ), and how to determine the function $H(\omega)$ and function $F(\omega)$ is shown by the following equations:

$$
\begin{aligned}
& H(\omega)=\int_{-\infty}^{\infty} h(t) e^{-i \omega t} \mathrm{~d} t, \\
& F(\omega)=\int_{-\infty}^{\infty} P(t) e^{-i \omega t} \mathrm{~d} t .
\end{aligned}
$$

The spectral density function $S_{w}(\omega)$ of the input response of the system is calculated by the frequency response function, and the spectral density function of the input $S_{f}(\omega)$ is calculated as the following:

$$
S_{w}(\omega)=|H(\omega)|^{2} S_{f}(\omega) .
$$

For the system of one degree, the frequency response function is

$$
H(\omega)=\frac{1}{-\omega^{2}+2 i \xi \omega_{0} \omega+\omega_{0}^{2}},
$$

in which $\omega_{0}, \xi$ is, respectively, the specific frequency and damper coefficient of the system. Similar to the system of more than one degree, the matrix of the frequency response is expressed as follows:

$$
[H(\omega)]=\frac{1}{-\omega^{2}[M]+i \omega[C]+[K]}=\sum_{j=1}^{n} \frac{\left\{\phi_{j}\right\}\left\{\phi_{j}\right\}^{T}}{\mu_{j}\left[\left(\omega_{j}^{2}-\omega^{2}\right)+2 i \xi_{j} \omega_{j}\right]},
$$

in which $[M],[C],[K]$, and $\{\phi\}$ are matrix of mass, damping, stiffness, and shape function of the system, respectively. The spectral density function $S_{w}(\omega)$ according to the density function equation (23) in statistical theory shows us the distribution of the boundary spectral amplitude values in the time domain. In the frequency domain, the spectral density function also shows that the energy distribution is satisfied. The definition of the spectrum central position is proposed to investigate the energy distribution characteristics of signals which do not satisfy in each frequency domain of the power spectral density. This concept introduces a sensitive parameter as a specific tool to identify characteristics of damage and monitor the structure status. While previous studies only define load-bearing objects, putting load effect under complete control, we developed this parameter based on the actual survey in the experiment. In addition, the study reckons the special features of the shift of central points in assessing the hardness reduction of the structure (see Figure 7). Suggestions for this new parameter include as follows:

(i) The CP-PSD can be calculated in the time domain, frequency domain, or both. Because the concept of the central point is quite flexible, responses in different measurement systems can be used. It brings an opportunity for application to status monitoring systems of discontinuous (nonperiodical fatigue forms) or continuous forcebearing structures (periodical fatigue forms).

(ii) The shift of this central position is quite suitable for both linear and nonlinear equations.

(iii) The CP-PSD provides energy information of the frequency range during the operation of the beam.

(iv) The CP-PSD does not depend on the vibrating phase effect (according to previous studies), thus preserves the important characteristics of the signal. Hence, 


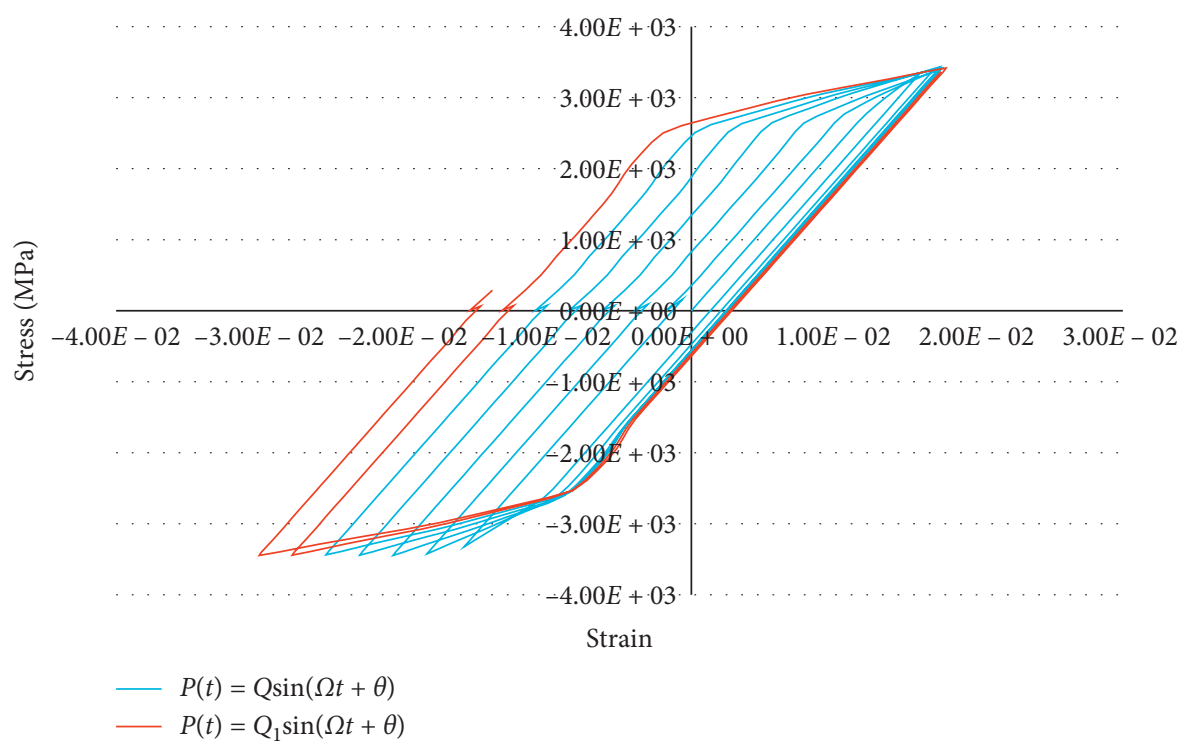

(a)

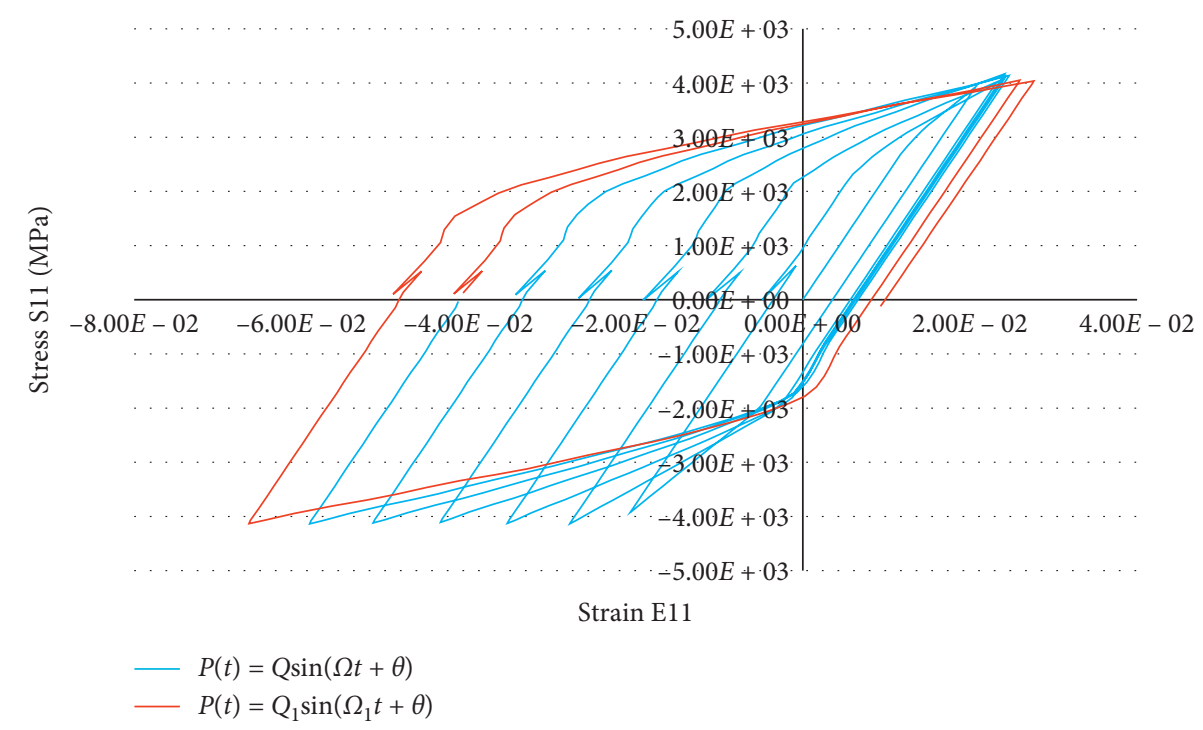

(b)

FIGURE 6: The hysteresis of 2 vibration amplitudes $Q$ and $Q_{1}$.

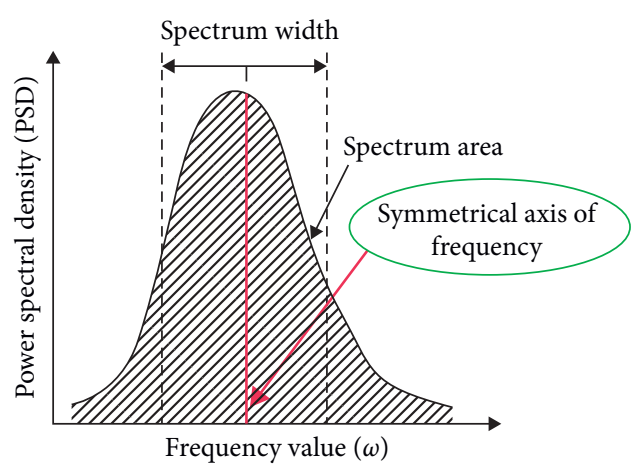

Figure 7: Determination of the central position of the representative power spectral density. the analysis does not have a significant effect on these changes.

\section{Conducting Experiment}

3.1. Experiment Model. In order to test in this study, we have built an experimental model generated at Faculty of Applied Science, Ho Chi Minh City University of Technology, as shown in Figure 8. The system consisted of major equipment: two-tip beam frame, forced-circulation vibration stimulating system, signal amplifier circuit, multichannel receiver circuit, computer, and signal wire. 


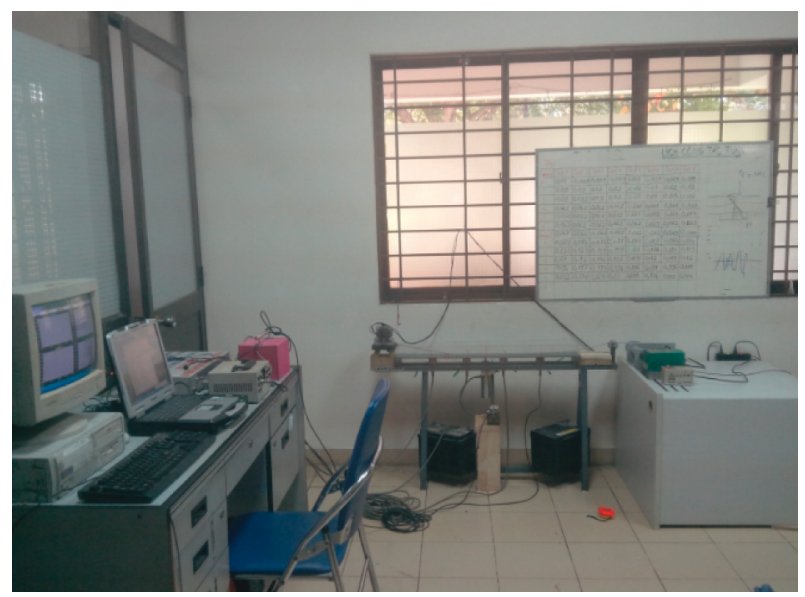

FIgURE 8: Arrangement of the experiment model.

\subsubsection{Clusters of the Experiment Model}

(1) Beam Structure. It consisted of a wooden beam of $1 \mathrm{~m}$ length, $10 \mathrm{~cm}$ width, and $1.5 \mathrm{~cm}$ thickness (see Figure 9) placed on two mounts as shown in Figure 10. According to Figure 10, experimental beams were mounted at both ends of the frame, and a vibration stimulating model as required by an inverter system as depicted in Figure 11 was placed in the middle of the beam. This stimulating system was adjusted based on 2 input parameters: vibration amplitude $(Q)$ and vibration frequency $(\Omega)$.

\subsubsection{Stimulating Cluster}

(1) Transmission System. It was composed by a belt transmission system in order to move the stimulating system on the beam model (see Figure 12). This system had transmission effects and movement orientation for the stimulating system. Thus, the system could move on beams at a steady speed without being impacted.

3.1.3. Signal Receiver Cluster. The signal receiver cluster which consisted of 4 measuring heads was implemented by an acceleration sensor. The measuring system was mounted under the beam and divided the beam length into 5 equal parts as depicted in Figure 13. These sensors transmitted signals through wires to a multichannel receiver to be stored on the host computer. The measurement signal acquisition diagram is shown in Figure 13.

3.2. Experimental Steps. The objective of the experiment was to investigate the reduction in bearing capacity of beams caused by fatigue effect. It was expressed by the number of forced-circulation periods on the beam. To meet that requirement, in this experiment, we used a beam made with plywood (medium-density fibreboard (MDF)). This is a soft material with high-fatigue bearing ability and wide application. The vibration of the beam under the impact of the stimulating model could easily respond to large amplitudes and high frequencies. Table 1 is the standard fatigue state of beams. There were 6 different

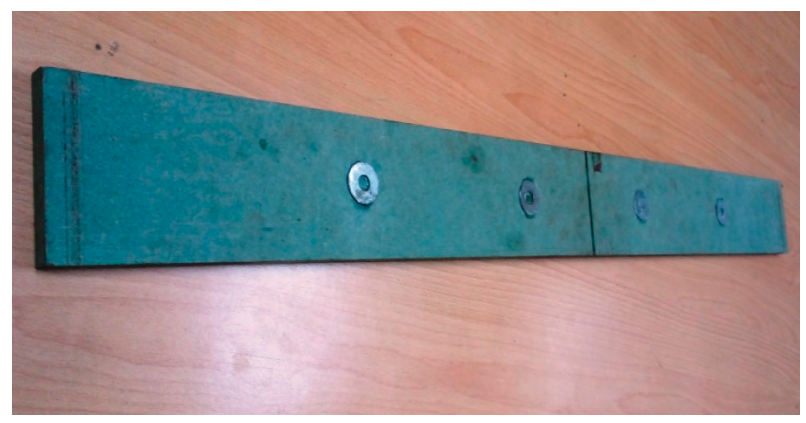

FIGURE 9: Fatigue test beam model.

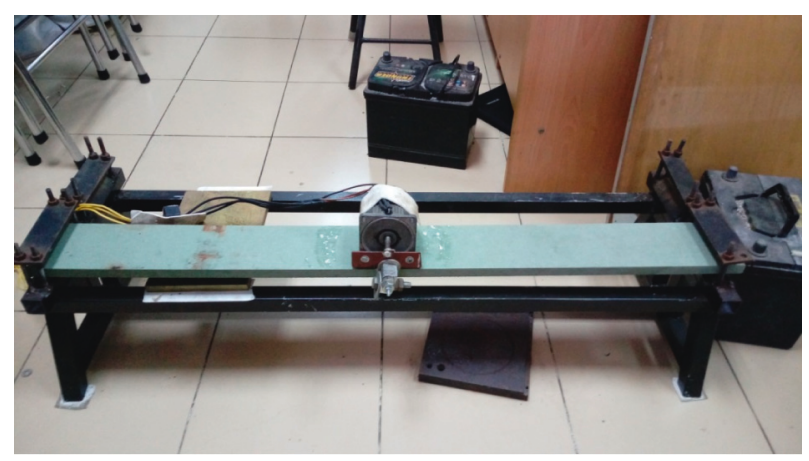

FIGURE 10: Experimental beams and the cuts.

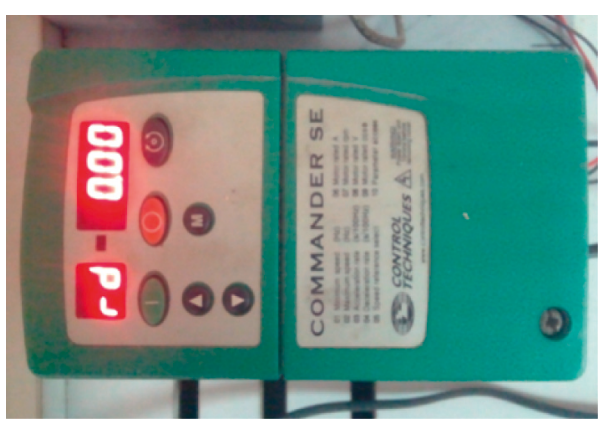

FIGURE 11: Inverter.

fatigue states corresponding to $10^{6}$ cycles ( $24 \mathrm{~h}$ of fatigue), and we investigated the mechanical state of the experimental beam to evaluate and identify its degradation level. The experiment was conducted in different levels of amplitude $\left(Q_{i}\right)$ and forced frequency $\left(\Omega_{i}\right)$. In this research, we only tested the fatigue position at the center of the beam without changing other positions. In the following studies, we will show the relationship between the position of fatigue and degradation level of the beam structure.

\section{Results and Discussion}

4.1. The Change of the Specific Frequency Value under the Influence of Fatigue. The specific frequency of the beam under the effect of the harmonic constraining force is determined by the power spectral density. The identifying method lets the beams vibrate freely under the effect of an instant force. The signal of the vibration amplitude obtained 


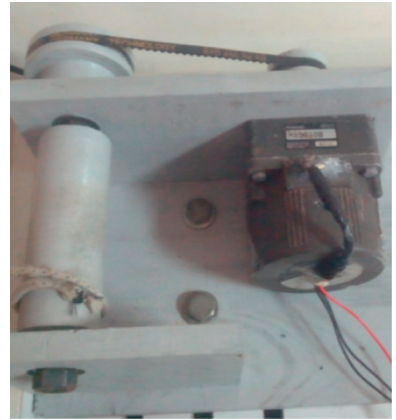

(a)

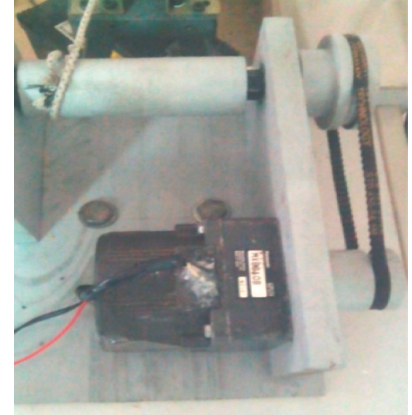

(b)

Figure 12: Transmission system.

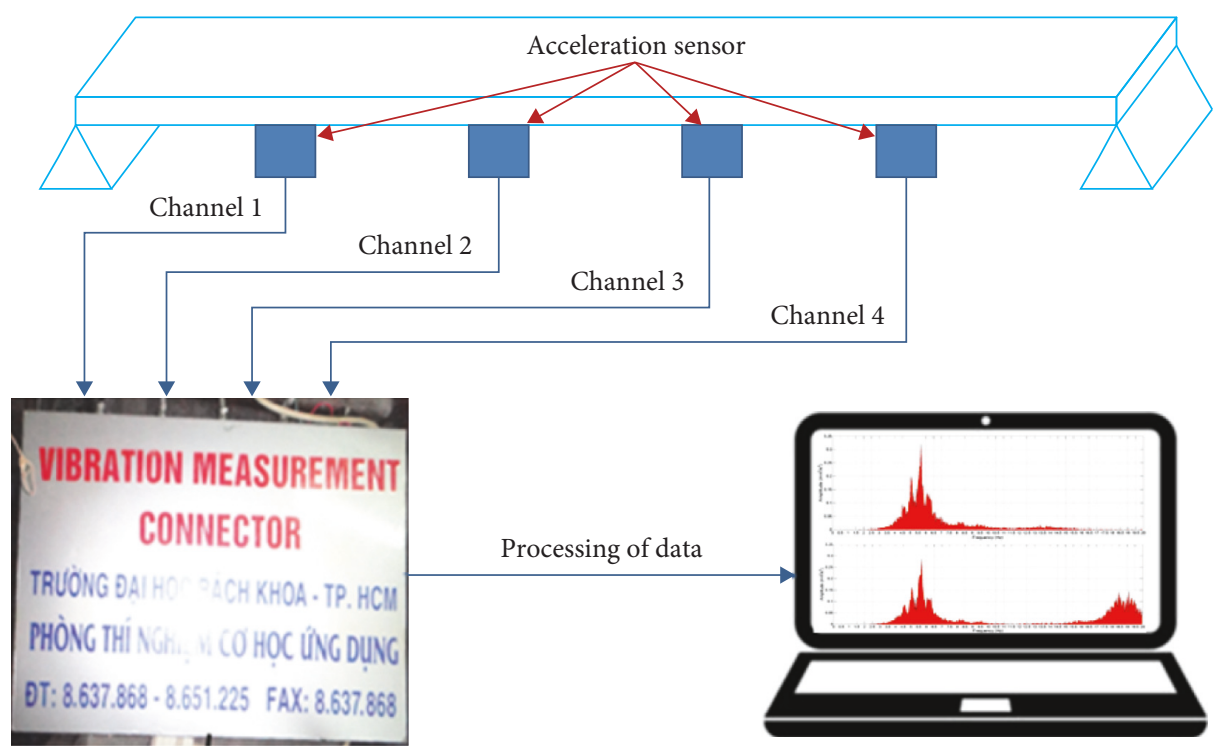

FIgURE 13: The signal received from the acceleration sensor.

TABle 1: Number of the vibration period.

\begin{tabular}{lcc}
\hline Symbol & Time (day) & Periods \\
\hline F1 & 0 & 0 \\
F2 & 1 & $10^{6}$ \\
F3 & 2 & $2 \times 10^{6}$ \\
F4 & 3 & $3 \times 10^{6}$ \\
F5 & 4 & $4 \times 10^{6}$ \\
F6 & 5 & $5 \times 10^{6}$ \\
\hline
\end{tabular}

from the acceleration sensors is shown in Figure 14, and the power spectral density generated from those sets whose shape is shown in Figure 15.

The experiment was conducted in 6 different fatigue states with an increasing period. The specific frequency values are obtained from Table 2, and the shape of the power spectral density corresponding to the fatigue states is shown in Figure 16.

Table 2 and Figure 16 show the following:

(i) Corresponding to 6 different fatigue states (from 0 to 6), the value of the frequency on the beam is almost unchanged $(0.5 \mathrm{~Hz})$ (according to Table 2).
The change is too small compared to the total number of fatigue periods on the beam. Thus, even though the parameter represents the reduction process of the bearing capacity of the structure, the frequency value is less sensitive than the requirements of identifying math.

(ii) Corresponding to 6 different fatigue states (from 0 to 6), the value of the frequency on the beam is almost unchanged $(0.5 \mathrm{~Hz})$ (according to Table 2). The change is too small compared to the total number of fatigue periods on the beam. Thus, even though the parameter represents the reduction process of the bearing capacity of the structure, the frequency value is less sensitive than the requirements of identifying math. The general shape of the power spectral density in 6 different fatigue states is quite similar. On the frequency domain, there are always two major domains $(3 \mathrm{~Hz}-27 \mathrm{~Hz})$ and $(27 \mathrm{~Hz}-60 \mathrm{~Hz})$. Thus, in terms of the mechanic behavior status of the beam under the effect of the fatigue period, there is only one form of vibration for all 6 states. 


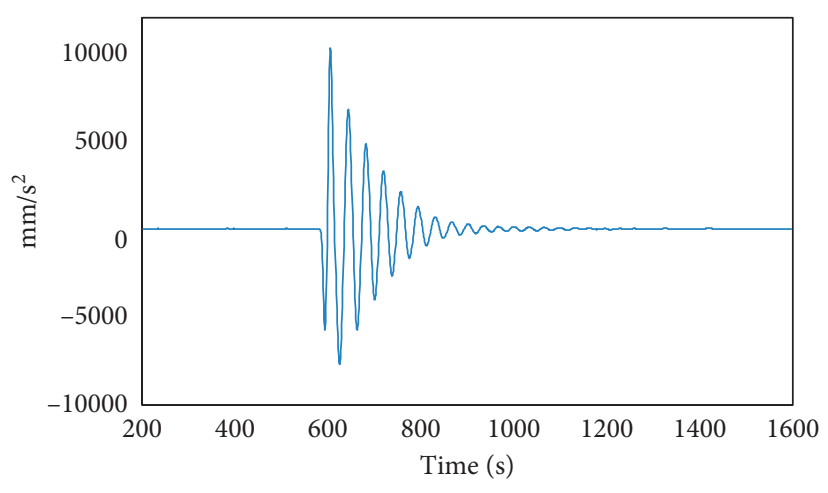

(a)

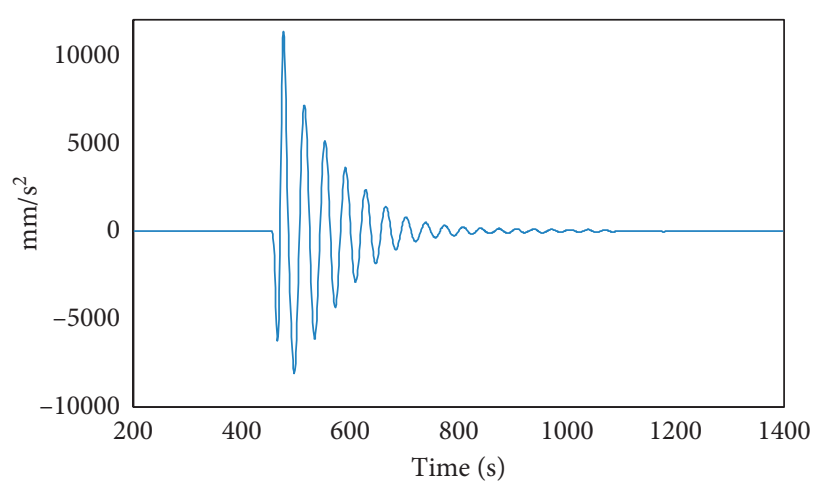

(b)

FIgURE 14: Signal amplitude of the beam vibration.

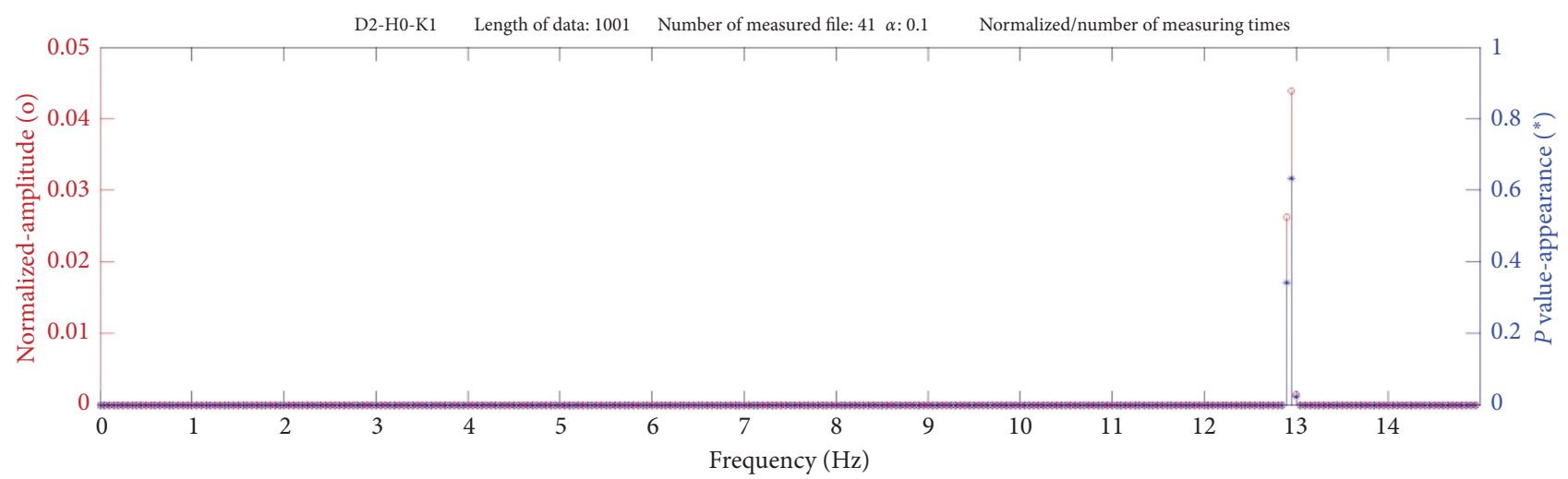

FIgURe 15: Power spectral density of freely vibrating beams.

TABle 2: The frequencies of beams during 6 fatigue cumulative periods.

\begin{tabular}{lcccccc}
\hline State & 1 & 2 & 3 & 4 & 5 & 6 \\
\hline Frequency $(\mathrm{Hz})$ & 13.8 & 13.8 & 13.7 & 13.6 & 13.5 & 13.3 \\
\hline
\end{tabular}

(iii) Corresponding to 6 different fatigue states (from 0 to 6), the value of the frequency on the beam is almost unchanged $(0.5 \mathrm{~Hz})$ (according to Table 2$)$. The change is too small compared to the total number of fatigue periods on the beam. Thus, even though the parameter represents the reduction process of the bearing capacity of the structure, the frequency value is less sensitive than the requirements of identifying math. In the two frequency domains of the beam, the second frequency domain $(27 \mathrm{~Hz}-60 \mathrm{~Hz})$ tends to change in magnitude (width) of the spectrum. As a general trend, the greater the state of fatigue is, the narrower and smaller the 2 nd frequency domain of the spectrum is. Therefore, to quantify the changing trend of the spectrum, the study proposes using the shift of the value in the central position of the spectrum.

4.2. Shifting the Central Position of the Power Spectral Density. As the degradation in bearing capacity of beam increases, it results in a decrease in the area of the high-frequency domain of the power spectral density as shown in Figure 16. In other words, the reduction of area 2 leads to a shift in the center (horizontal) of the whole spectrum. In order to quantify this shift, this paper proposes using the concept of 7 centers as shown in Figure 17. In the first step, the whole area is divided into two equal areas by the fourth central (the center in 7 selected central). The two small areas divided at step 1 continue to be divided by two centers 2 and 6. In Step 2 , the spectrum is divided into four areas by centers 2,4 , and 6. Those areas are divided into two by centers $1,3,5$, and 7 , depicted in Figure 17. Such division makes the variable frequency areas of the power spectrum equal. This makes the centers of spectral areas more sensitive to changes in fatigue states. In theory, it is possible to divide even smaller areas to examine the change of values in the central point. However, splitting too much makes the value of the area scattered, which makes it more difficult to determine change of the spectrum. When we applied the same identifying method for the experimental model, the survey conducted 4 measurement channels corresponding to 4 different measurement positions. The results are expressed as Figures 18(a)-18(d).

To be more visual in the evaluation process, this study uses a line graph to show shifts. By standard, 7 lines on the graph that show the shift of the central point are arranged in order from low to high (the central point lines from 1 to 7 ). This is the line that shows the central of the power spectral density through 6 different fatigue states of forced-circulation 


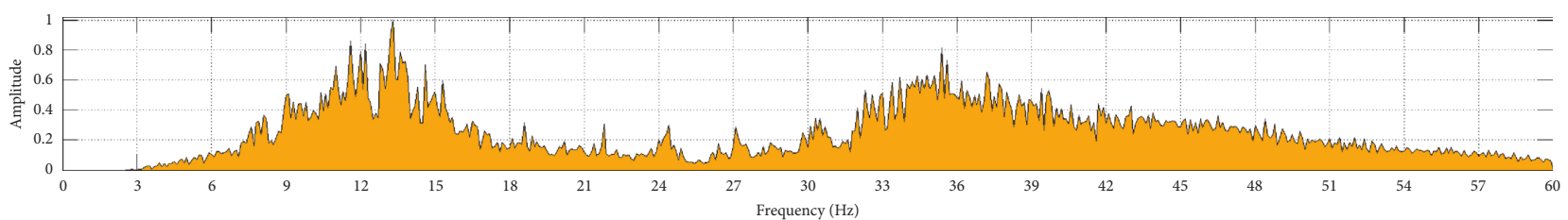

(a)

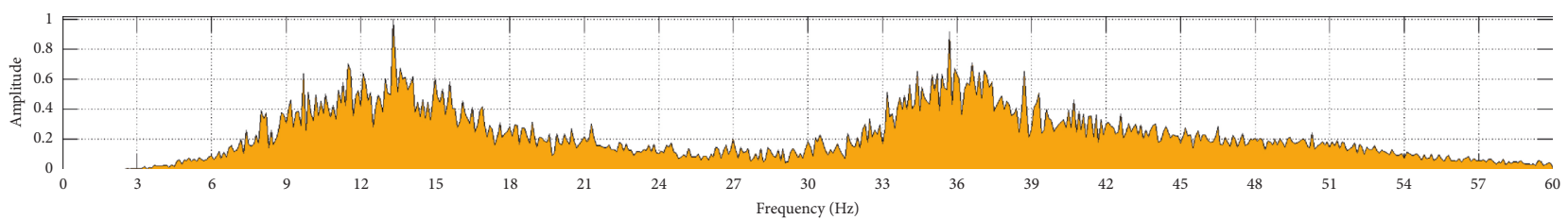

(b)

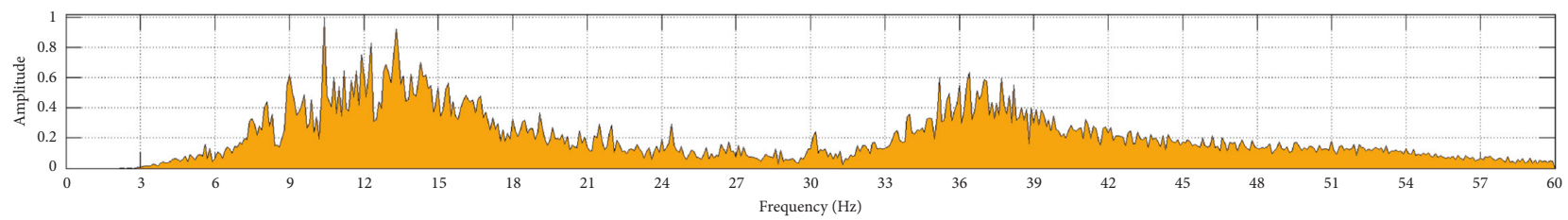

(c)

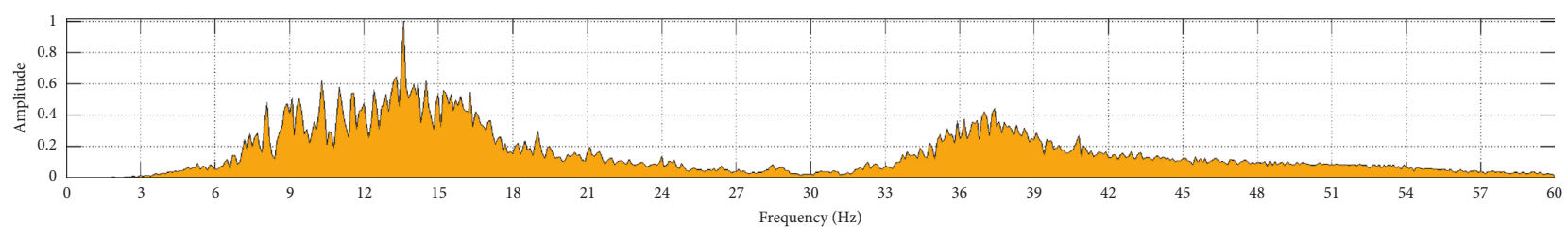

(d)

Figure 16: Power spectral density of beams corresponding to different fatigue states.

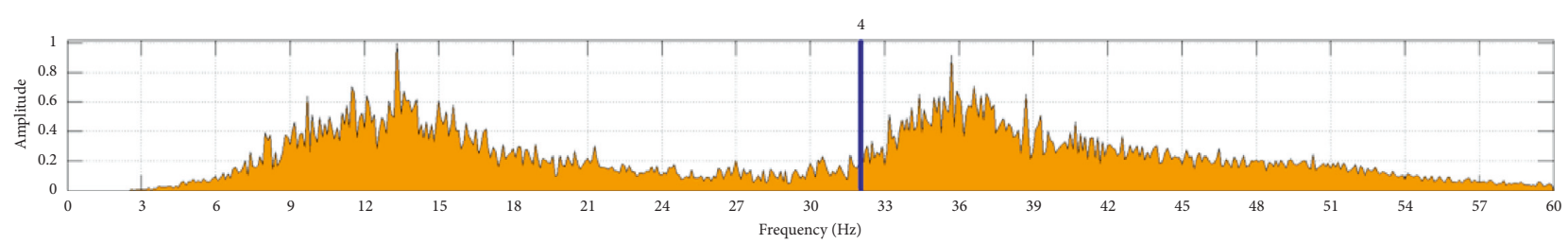

(a)

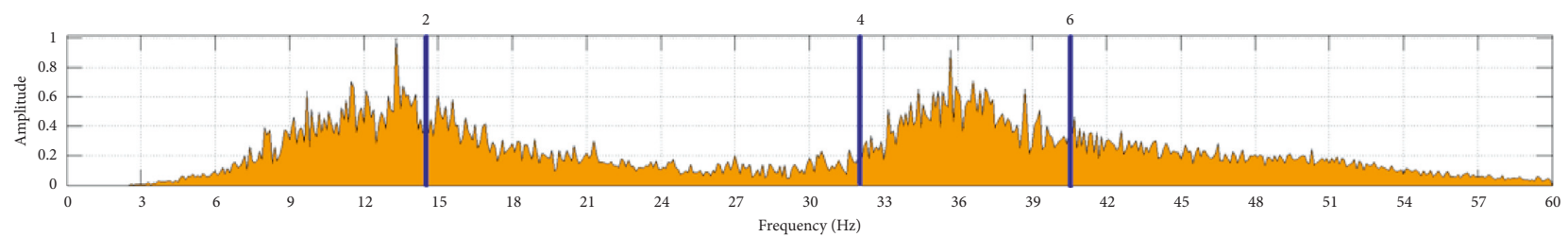

(b)

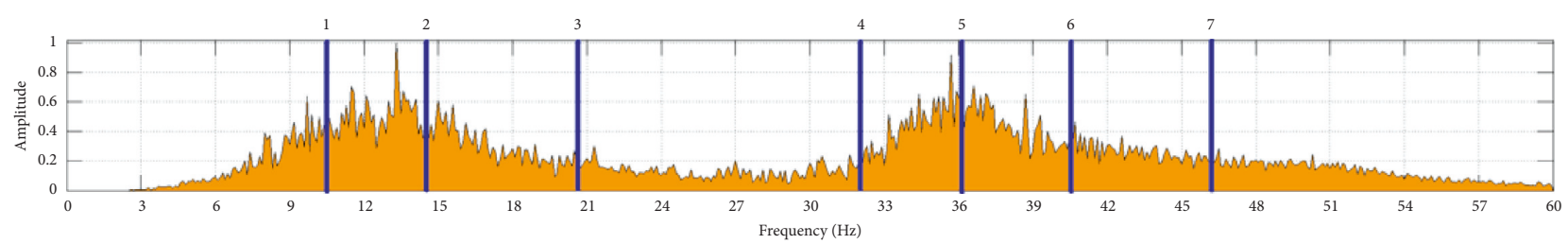

(c)

FIGURE 17: Steps to identify 7 centers. 

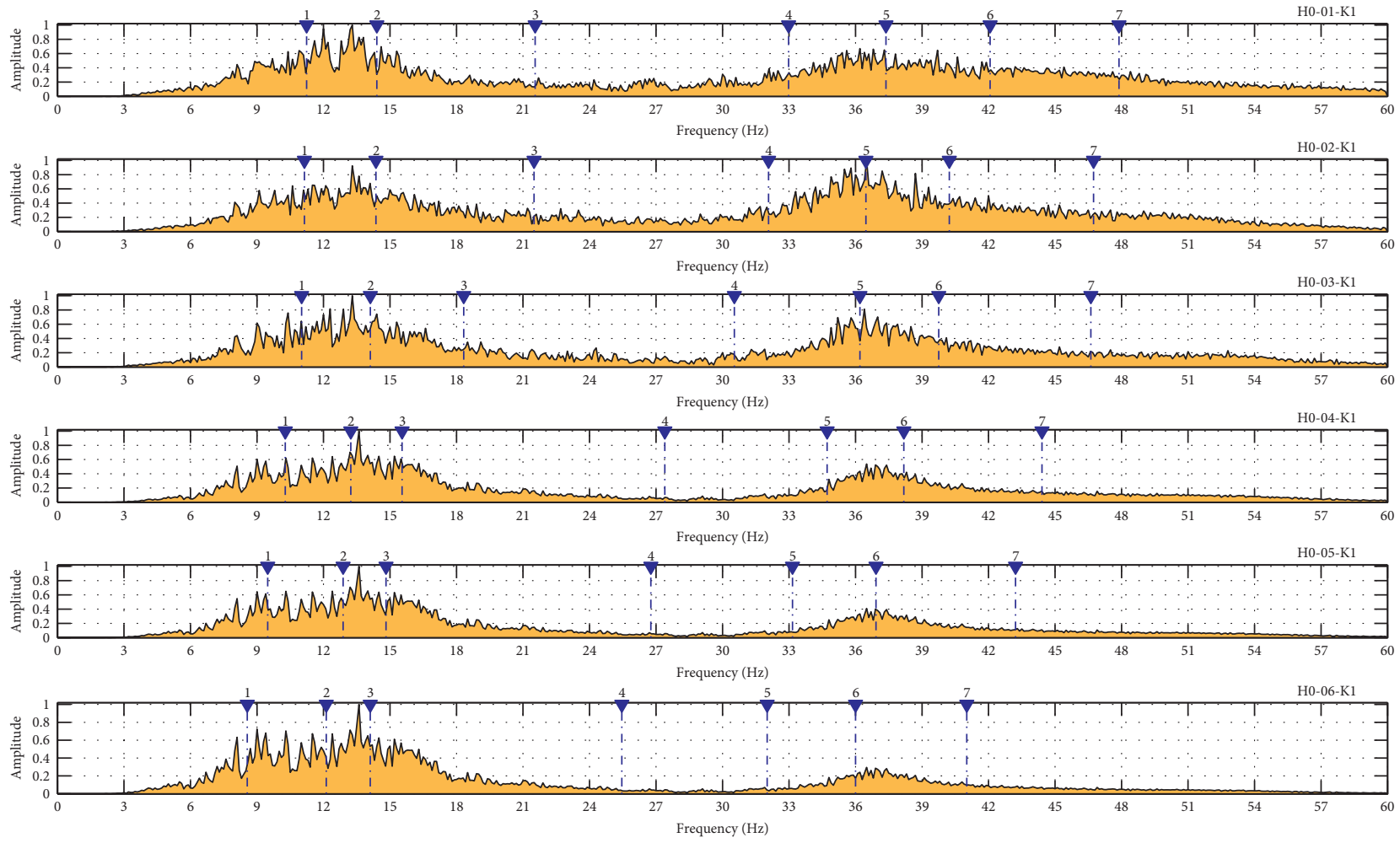

(a)
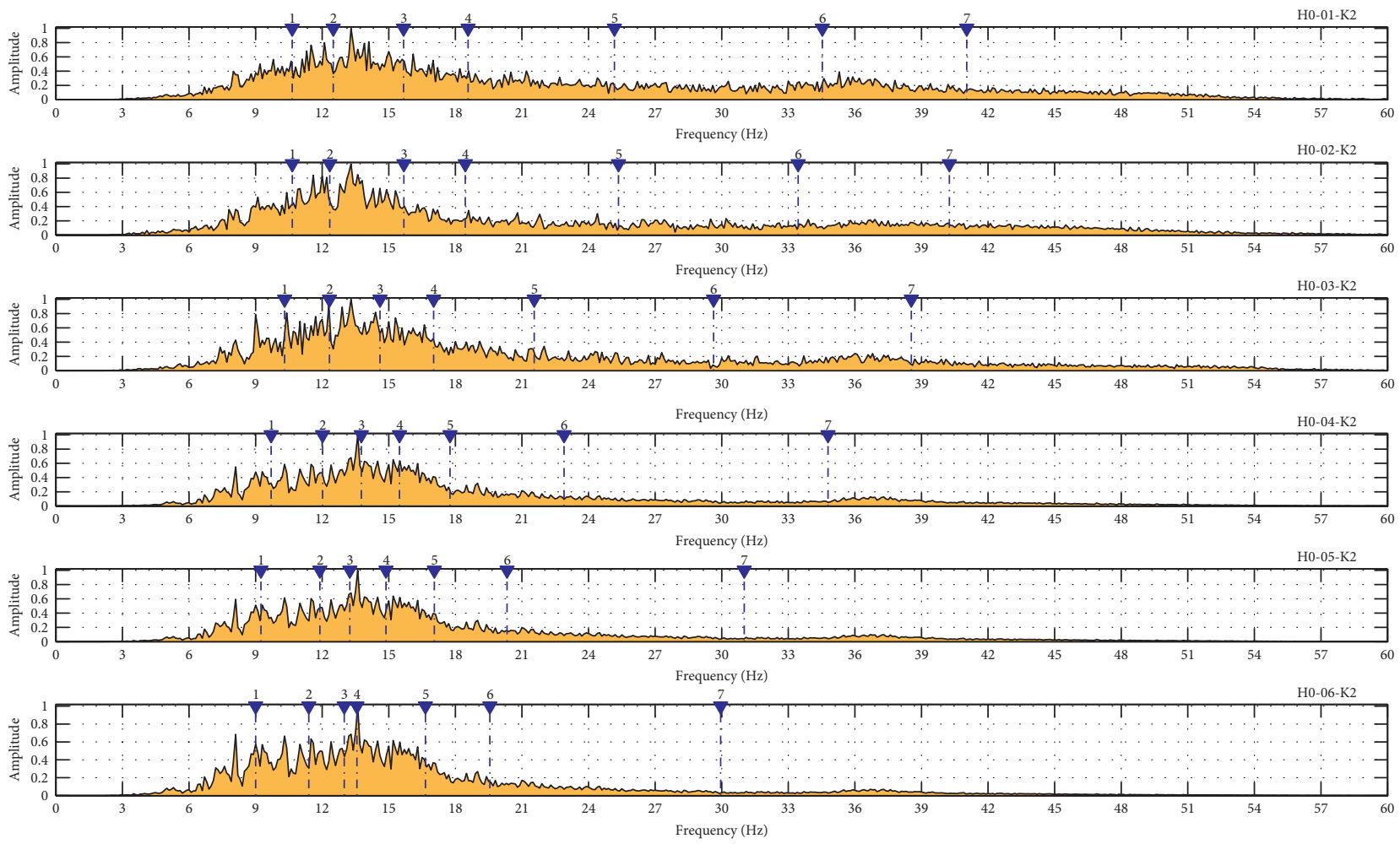

(b)

Figure 18: Continued. 

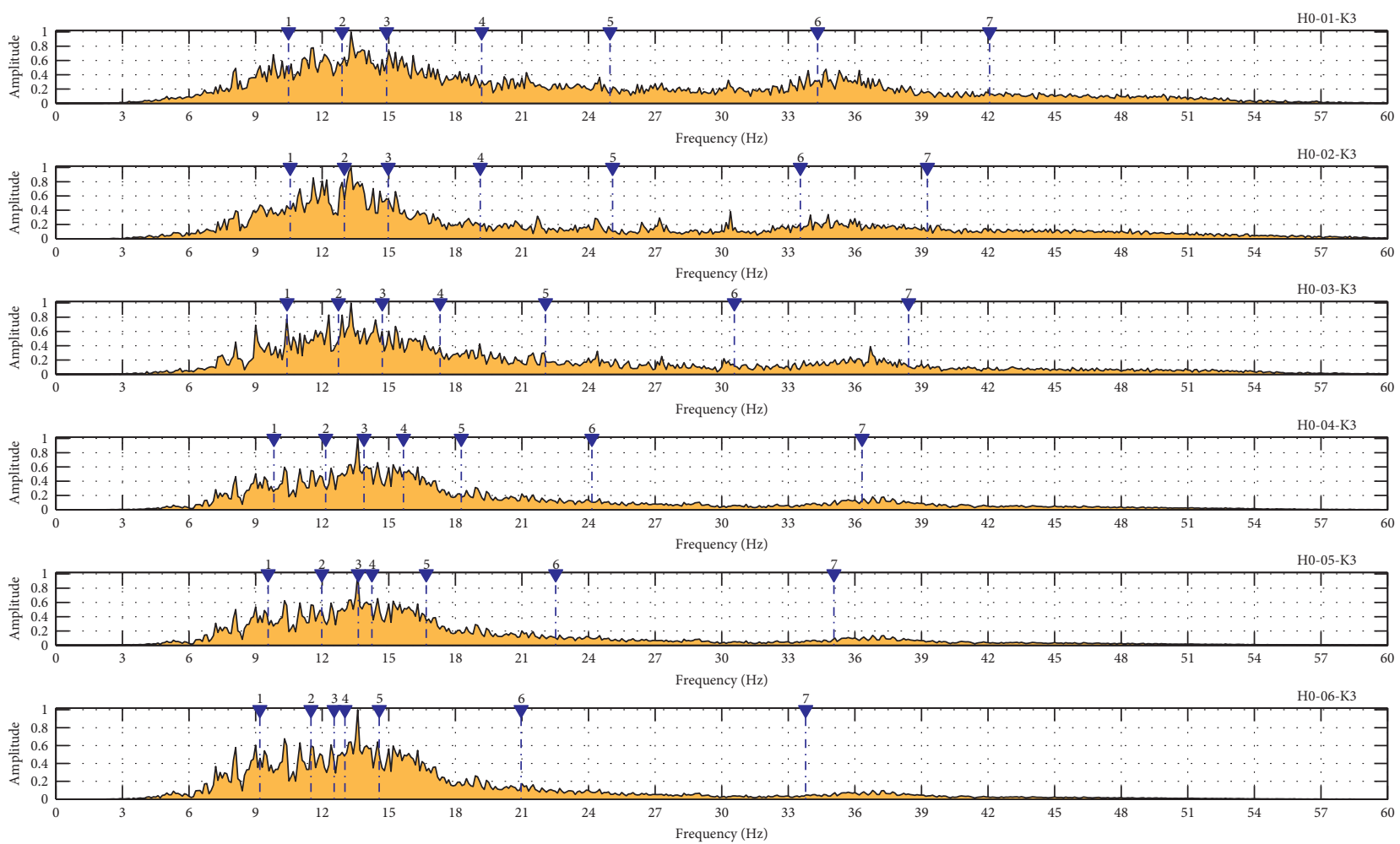

(c)
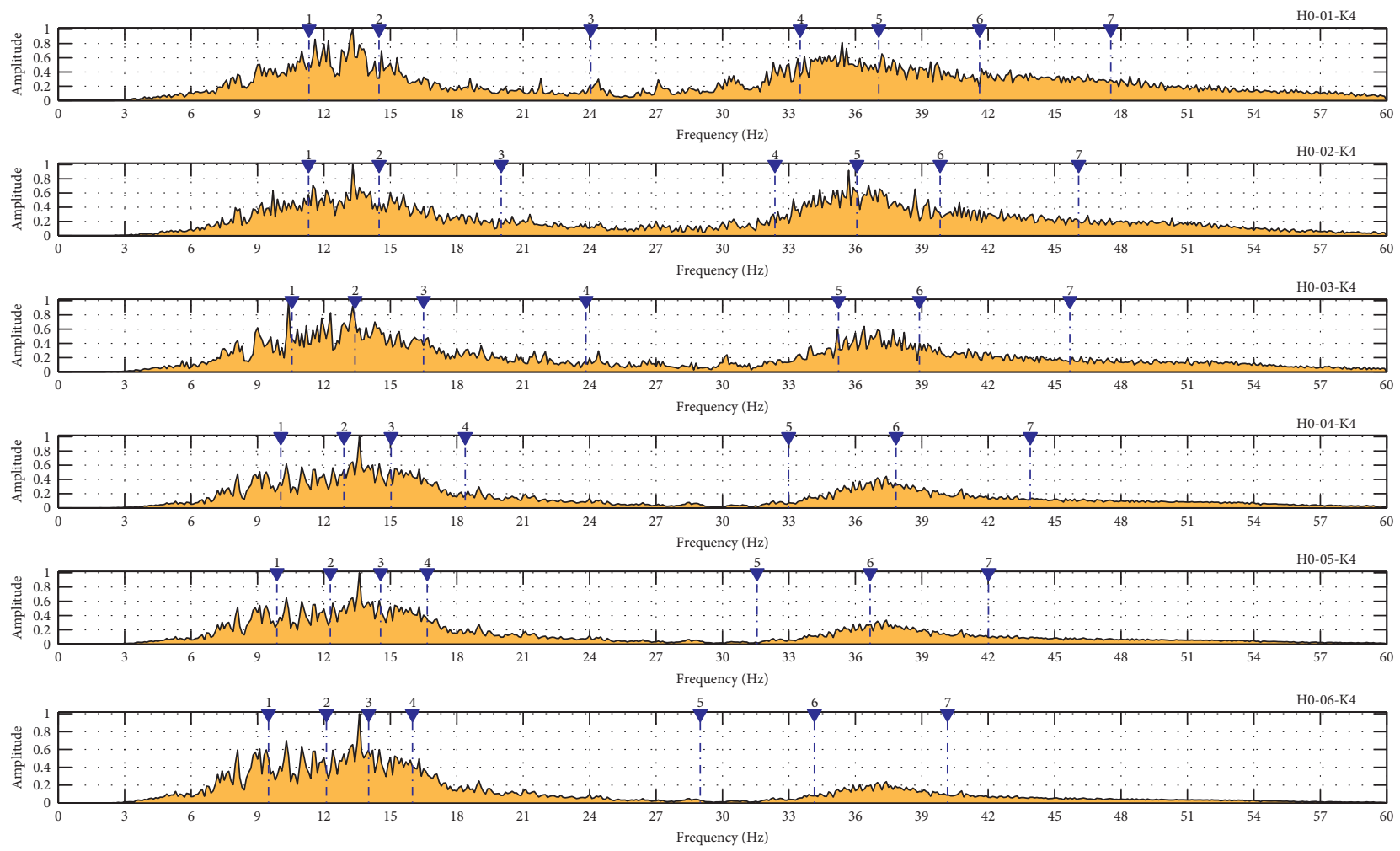

(d)

FIGURE 18: The change of the central position of the spectrum through 4 measurement channels of 6 different fatigue states. 

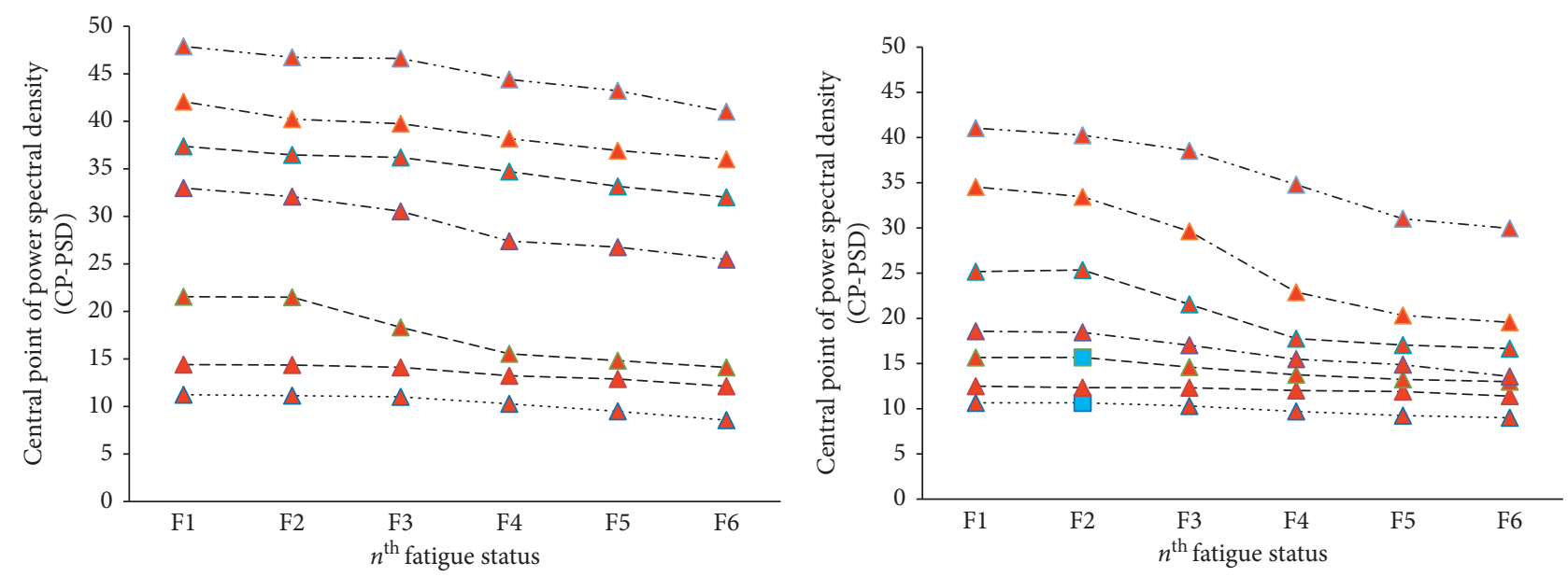

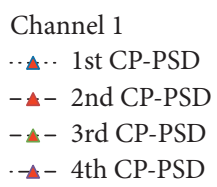

- \pm-4 th CP-PSD

(a)

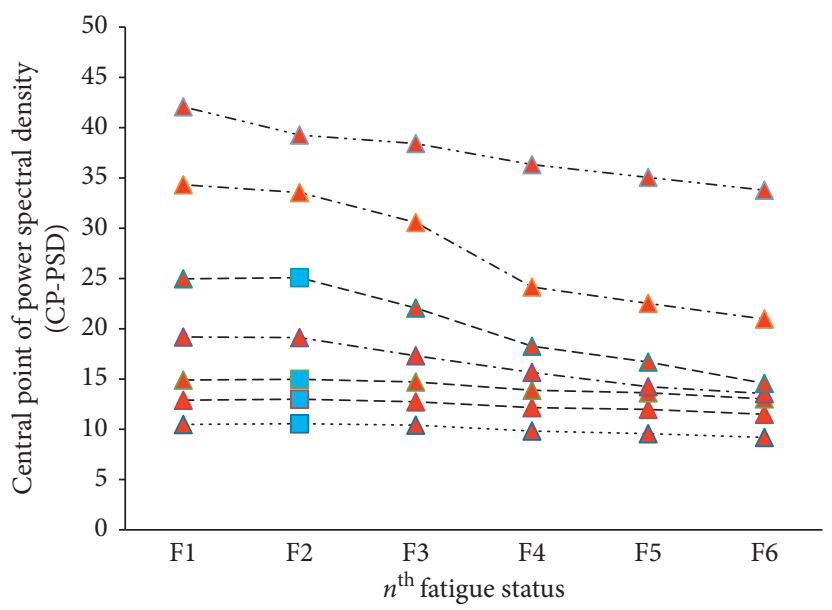

Channel 3

-... 1st CP-PSD

- -2 2nd CP-PSD

- -3 rd CP-PSD

-4 th CP-PSD

(c)

$$
\begin{aligned}
& \text { - }-5 \text { th CP-PSD } \\
& \text { - }-6 \text { th CP-PSD } \\
& \text {.... 7th CP-PSD }
\end{aligned}
$$

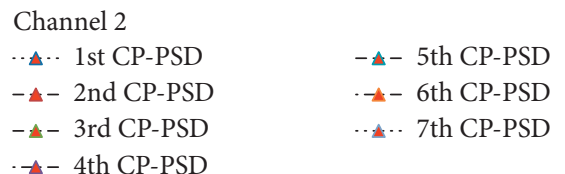

(b)

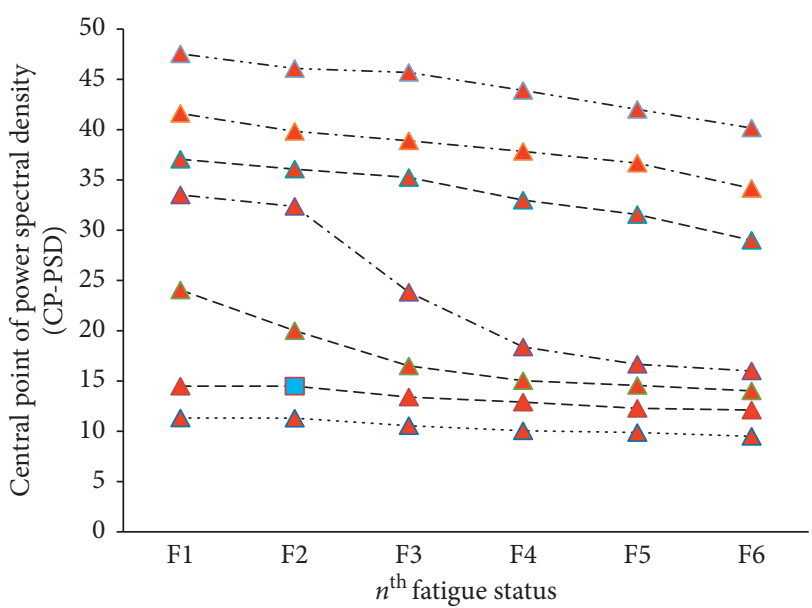

Channel 4

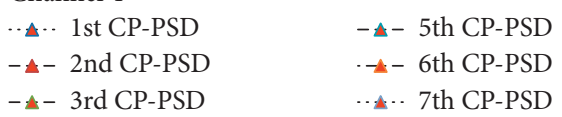

-4 th CP-PSD

(d)

Figure 19: Line graphs showing the position of 7 central points of beams with 4 fatigue levels.

vibration on the beam. We also agree that the points representing the reduced value are marked by $\Delta$ (if the frequency value at the point is lower than the preceding point) and $\square$ in the opposite case.

According to Figure 19, the general trend of the lines representing the center of the spectrum is going down. In other words, the centers tend to move to the left, which means that the value decreases gradually by different fatigue periods. It indicates that corresponding to the decline of the specific frequency according to Table 2, the value of the CP-PSD also decreases. However, the decline level of the central position is more rapid and clearer than the specific frequency value. In the experiment, there was an increase at some central points compared to the previous fatigue state, but the increase was negligible (below 1\%), and the number of increasing points was less than the total points on each graph. In addition, due to the arrangement at four measuring channels, we also find symmetry in terms of spectral shape and central value. Accordingly, channels 1 and 4 are the same. They tend to be higher than channels 2 and 3 in terms of amplitude values. This is the basis for quantifying the position when a defect is detected on the beam under harmonic constraining force.

The distinctive feature of the central shift allows us to get clearer assessment in each state of the fatigue period when 


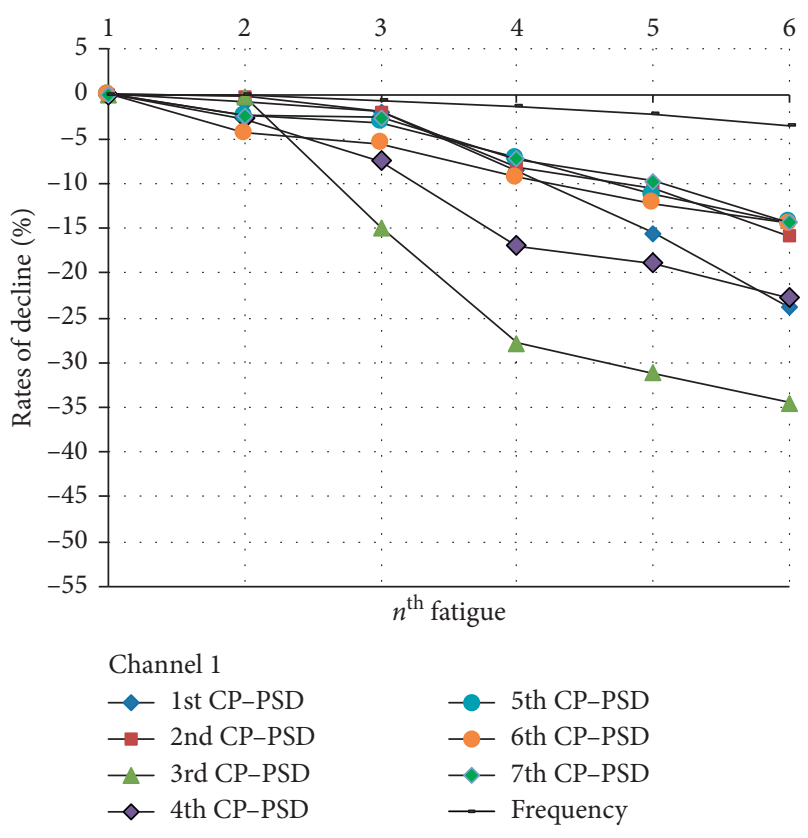

FIgURE 20: The declined rates in channel 1 with 7 central points in 4 fatigue levels.
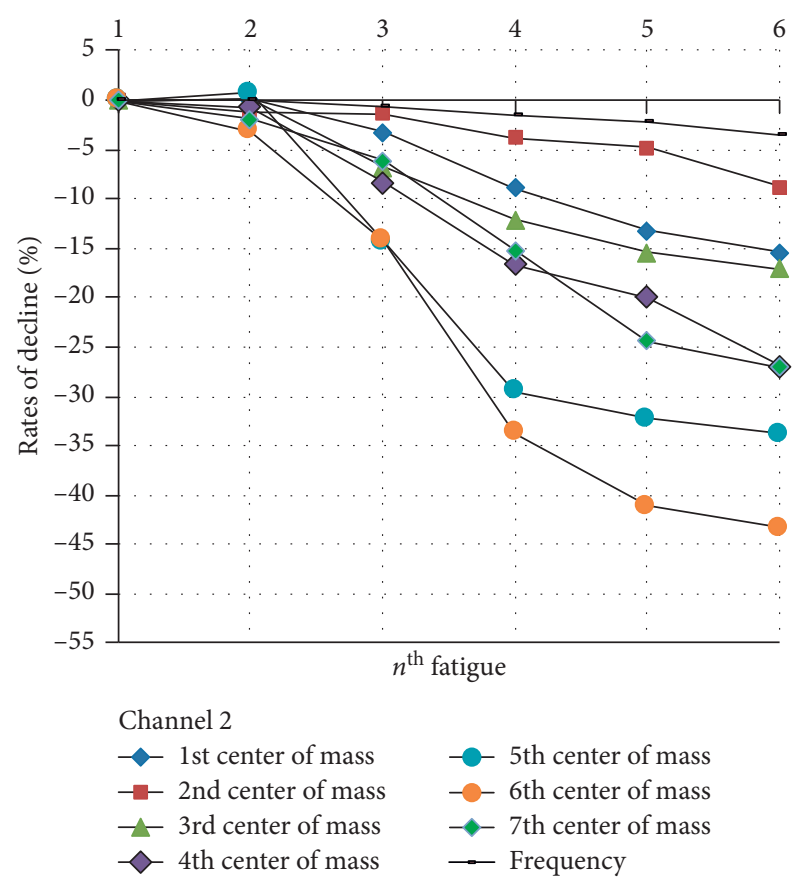

FIGURE 21: The declined rates in channels 2 with 7 central points in 4 fatigue levels.

compared to the change of specific frequency value. Accordingly, there are states where the specific frequency remains constant (from fatigue state 1 to 2 ); however, the value of the central point still shows a shift to the left (decline in value). Thus, in states where the specific frequency has not changed yet, in fact, it still has a decrease in energy, especially in high-frequency areas. This energy degradation must reach a certain level for the new frequency to continue to decrease.

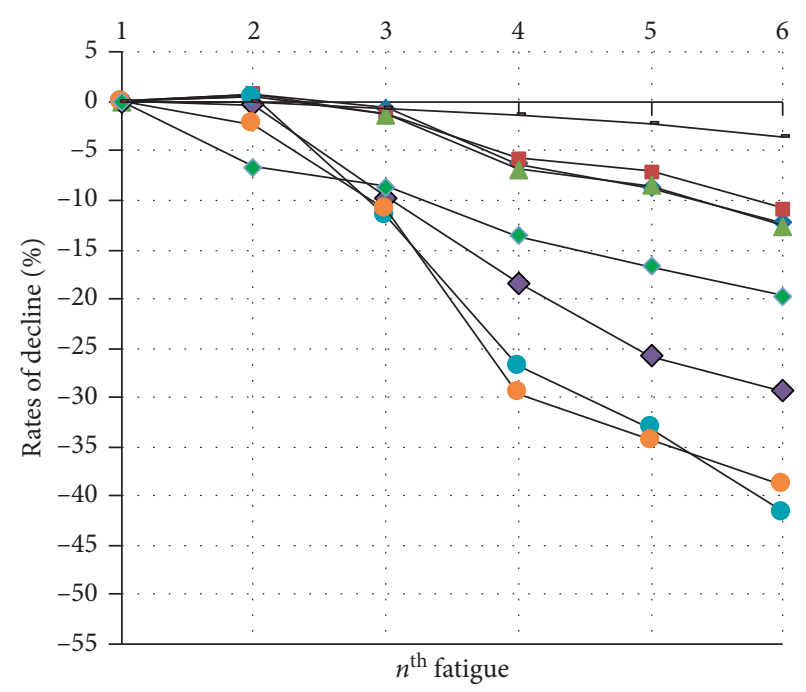

Channel 3

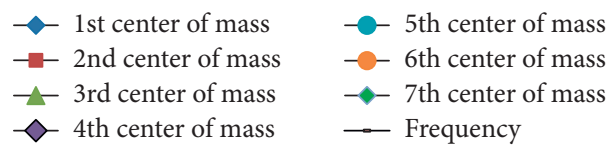

FIGURE 22: The declined rates in channels 3 with 7 central points in 4 fatigue levels.

However, the central value represents distribution of energy on the spectrum, and with only a small change, this value can be shifted. In brief, this value, which is a new parameter, has much higher sensitivity than others.

4.3. The Sensitivity Comparison of the Change of Spectrum's Central Position and Specific Frequency Value. The comparison of the decrease level at the central position with a specific frequency expressed is shown in Figures 20-23.

Figures 20-23 show that central points experience greater change than their specific frequency value. Specifically, for beams from the original state to the $6^{\text {th }}$ fatigue phase (with $6 \times 10^{6}$ fatigue cycles), the specific frequency values only change $3.6 \%$. Meanwhile, the central position value changes at least $8.8 \%$ (the second central position of the power spectral density according to channel 4) and up to $52.2 \%$ (the fourth central position according to channel 4).

The distribution of central position's characteristics of the power spectral density is different at each point. In particular, the distribution of channels 1 and 4 is similar and that of the channels 2 and 3 is similar. Based on this factor, we can see that the degradation on beams is uneven at different points. Therefore, this parameter can be applied to identify the most degrading points of the tested beams. In contrast, the specific frequency values at different points are equal. Therefore, the distribution of energy in terms of mechanics expressed by its own specific frequency value does not fully reflect the dispersion process. The forced circulation is the cause of fatigue on beams. However, the energy dispersion is not big enough for the specific frequency value to decrease quickly. Thus, the central position 


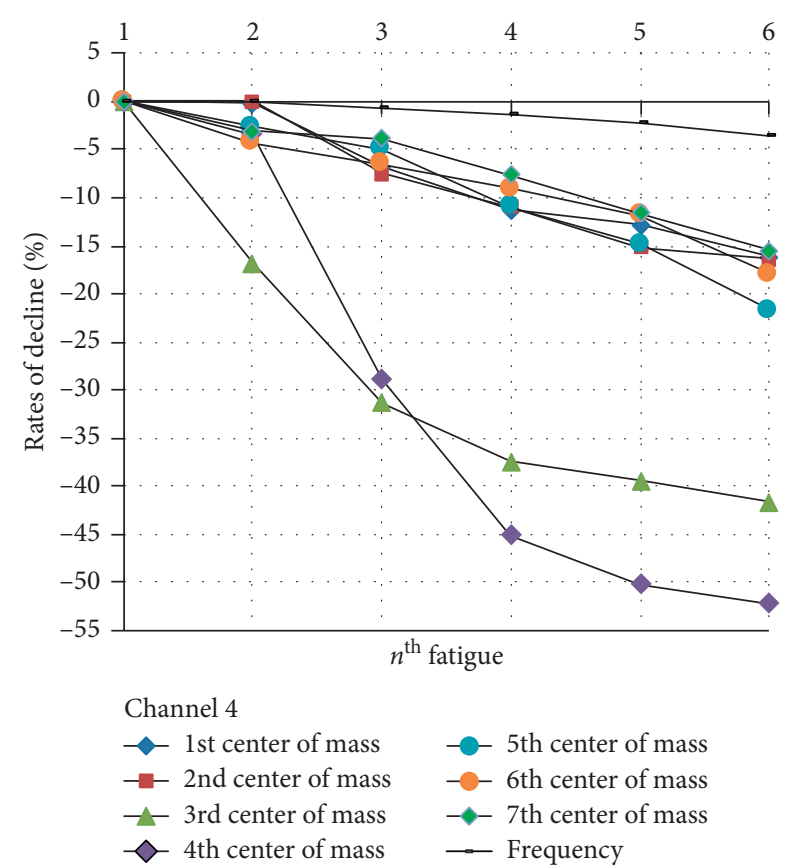

FIGURE 23: The declined rates in channels 4 with 7 central points in 4 fatigue levels.

value of the power spectral density shows the process of dissipating energy on the beam in this experiment.

Central points $3 \mathrm{rd}$, 4th, 5th, 6th, and 7th experience greater changes than 1 st and $2 \mathrm{nd}$. This indicates that there is a rapid change in the area with the higher frequency domain (the second frequency range varies from $27 \mathrm{~Hz}$ to $60 \mathrm{~Hz}$ ). Conversely, this value moves less in the lower frequency domain. This also confirms the sensitivity of 1 st area in comparison to 2 nd area. On the contrary, frequency value of the first area usually contains the value of the specific frequency on the structure. All in all, this is the main cause of the nonsensitivity of specific frequency values in this study as well as in other authors' studies.

\section{Conclusion}

The above results show the CP-PSD distribution of the vibrating signal is more sensitive than the specific frequency. This is a new parameter to assess the reduction of bearing capacity of the beam under the effect of periodically forced circulation. The sensitivity of the parameter is expressed by not only the decrease rate in each measurement channel but also the level of degradation on the tested beam. The results show that even when the specific frequency value is constant, the central points still have a certain shift. It means the new parameters are sensitive enough with small changes in the mechanical characteristics of materials when causing fatigue on beams. In addition, the distribution of central position value of the power spectral density indicates the degradation of the beam at different positions. In the future, the authors will quantify the degradation level of the beam corresponding with different fatigue cycles. This is the premise to assist evaluation of the structure lifespan under the effect of fatigue.

\section{Data Availability}

All data generated or analysed during this study are included in this work.

\section{Conflicts of Interest}

The authors declare that they have no conflicts of interest.

\section{Acknowledgments}

This research was funded by Ho Chi Minh City University of Technology, VNU-HCM, under grant number BK-SDH2019-1880703.

\section{References}

[1] F. M. Burdekin and D. E. W. Stone, "The crack opening displacement approach to fracture mechanics in yielding materials," Journal of Strain Analysis, vol. 1, no. 2, pp. 145-153, 1966.

[2] J. W. Hutchinson, "Singular behaviour at the end of a tensile crack in a hardening material," Journal of the Mechanics and Physics of Solids, vol. 16, no. 1, pp. 13-31, 1968.

[3] J. R. Rice and G. F. Rosengren, "Plane strain deformation near a crack tip in a power-law hardening material," Journal of the Mechanics and Physics of Solids, vol. 16, no. 1, pp. 1-12, 1968.

[4] C. F. Shih, "Relationships between the J-integral and the crack opening displacement for stationary and extending cracks," Journal of the Mechanics and Physics of Solids, vol. 29, no. 4, pp. 305-326, 1981.

[5] K. Pereira, S. Bordas, S. Tomar et al., "On the convergence of stresses in fretting fatigue," Materials, vol. 9, no. 8, p. 639, 2016.

[6] D. Kumar, R. Biswas, L. H. Poh, and M. A. Wahab, "Fretting fatigue stress analysis in heterogeneous material using direct numerical simulations in solid mechanics," Tribology International, vol. 109, pp. 124-132, 2017.

[7] K. Pereira, T. Yue, and M. Abdel Wahab, "Multiscale analysis of the effect of roughness on fretting wear," Tribology International, vol. 110, pp. 222-231, 2017.

[8] T. Yue and M. Abdel Wahab, "A numerical study on the effect of debris layer on fretting wear," Materials, vol. 9, no. 7, p. 597, 2016.

[9] T. Yue and M. Abdel Wahab, "Finite element analysis of fretting wear under variable coefficient of friction and different contact regimes," Tribology International, vol. 107, pp. 274-282, 2017.

[10] T. Yue and M. Abdel Wahab, "Finite element analysis of stress singularity in partial slip and gross sliding regimes in fretting wear," Wear, vol. 321, pp. 53-63, 2014.

[11] O. Vingsbo and S. Söderberg, "On fretting maps," Wear, vol. 126, no. 2, pp. 131-147, 1988.

[12] N. A. Bhatti and M. Abdel Wahab, "Fretting fatigue damage nucleation under out of phase loading using a continuum damage model for non-proportional loading," Tribology International, vol. 121, pp. 204-213, 2018.

[13] N. A. Bhatti and M. Abdel Wahab, "Fretting fatigue crack nucleation: a review," Tribology International, vol. 121, pp. 121-138, 2018.

[14] W. N. Findley, J. J. Coleman, and B. C. Handley, "Theory for combined bending and torsion fatigue with data for SAE 4340 steel," Technical Report, Engineering Materials Research Laboratory, Brown University, Providence, RI, USA, 1956. 
[15] W. N. Findley, "A theory for the effect of mean stress on fatigue of metals under combined torsion and axial load or bending," Journal of Manufacturing Science and Engineering, vol. 81, no. 4, pp. 301-305, 1959.

[16] D. L. McDiarmid, "A general criterion of high cycle multiaxial fatigue," Fatigue \& Fracture of Engineering Materials \& Structures, vol. 14, no. 4, pp. 429-453, 2007.

[17] C. D. Lykins, S. Mall, and V. K. Jain, "A shear stress-based parameter for fretting fatigue crack initiation," Fatigue, vol. 24, no. 7, pp. 461-473, 2001.

[18] F. Ali and D. F. Socie, "A critical plane approach to multiaxial fatigue damage including out-of-phase loading," Fatigue \& Fracture of Engineering Materials \& Structures, vol. 11, no. 3, pp. 149-165, 1988.

[19] K. Smith, T. Topper, and P. Watson, "A stress-fatigue function for the fatigue of metals," Journal of Materials Science, vol. 5, pp. 767-778, 1970.

[20] M. P. Szolwinski and T. N. Farris, "Mechanics of fretting fatigue crack formation,” Wear, vol. 198, no. 1-2, pp. 93-107, 1996.

[21] Y. Zhou and J. Tao, "Theoretical and numerical investigation of stress mode shapes in multi-axial random fatigue," $\mathrm{Me}$ chanical Systems and Signal Processing, vol. 127, pp. 499-512, 2019.

[22] Y. Zhou, S. Wu, N. Trisovic, Q. Fei, and Z. Tan, "Modal strain based method for dynamic design of plate-like structures," Shock and Vibration, vol. 2016, Article ID 2050627, 10 pages, 2016.

[23] Y. Zhou, Q. Fei, and S. Wu, "Utilization of modal stress approach in random-vibration fatigue evaluation," Proceedings of the Institution of Mechanical Engineers Part G Journal of Aerospace Engineering, vol. 231, no. 14, 2016. 


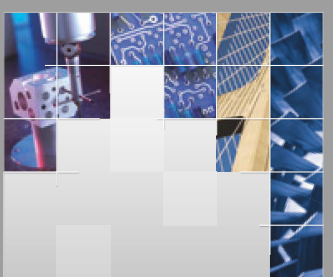

\section{Enfincering}
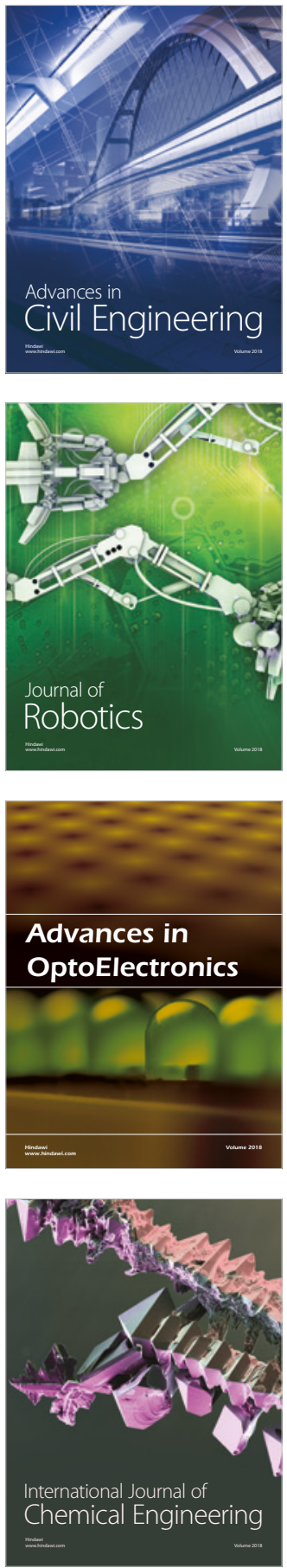

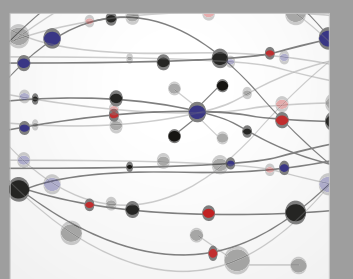

\section{Rotating \\ Machinery}

The Scientific World Journal

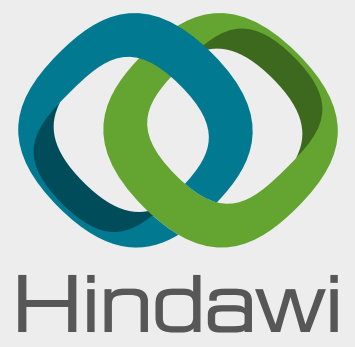

Submit your manuscripts at

www.hindawi.com
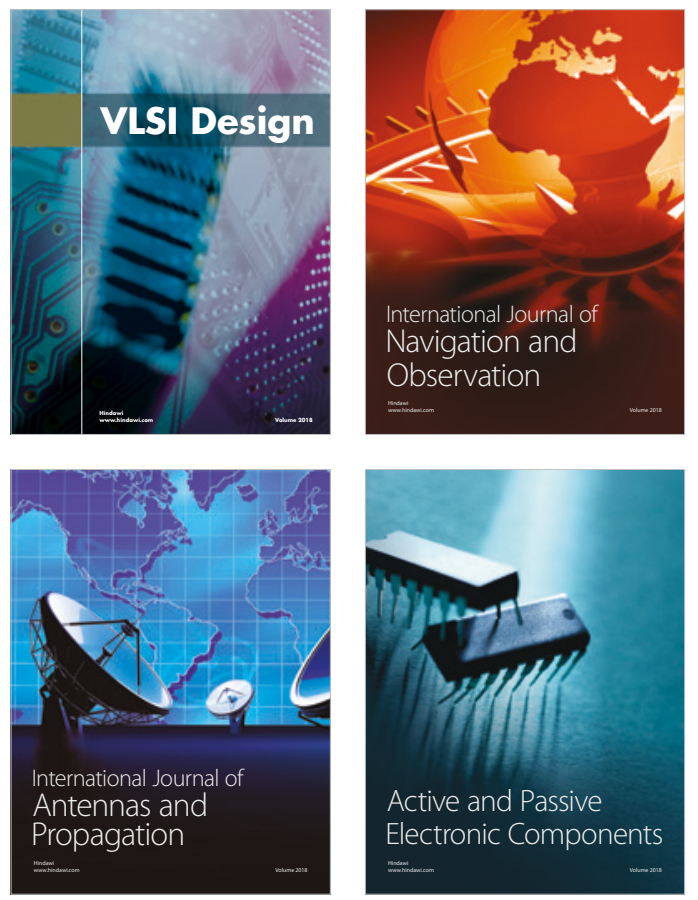
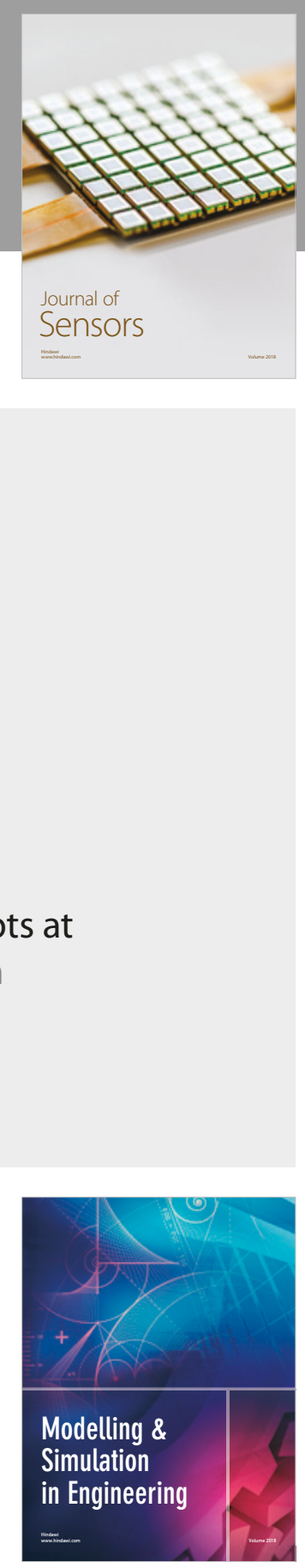

\section{Advances \\ Multimedia}
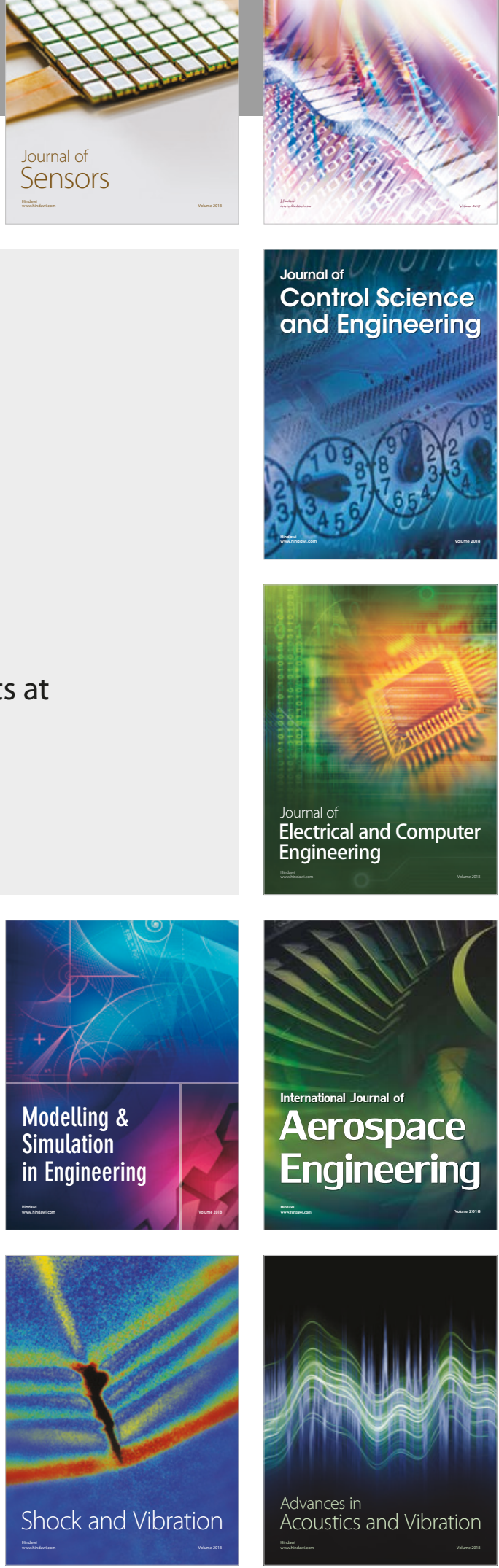